\title{
Joint Functional Calculus for Definitizable Self-adjoint Operators on Krein Spaces
}

\author{
Michael Kaltenbäck@ and Nathanael Skrepek
}

Dedicated to Heinz Langer on the occasion of his 85th birthday

\begin{abstract}
In the present note a spectral theorem for a finite tuple of pairwise commuting, self-adjoint and definitizable bounded linear operators $A_{1}, \ldots, A_{n}$ on a Krein space is derived by developing a functional calculus $\phi \mapsto \phi\left(A_{1}, \ldots, A_{n}\right)$ which is the proper analogue of $\phi \mapsto \int \phi d E$ in the Hilbert space situation with the common spectral measure $E$ for a finite tuple of pairwise commuting, self-adjoint bounded linear operators.
\end{abstract}

Mathematics Subject Classification. 47A60, 47B50, 47B15.

Keywords. Krein space, Definitizable operators, Self-adjoint operators, Spectral theorem.

\section{Introduction}

In the Hilbert space setting the spectral theorem for bounded linear, selfadjoint operators is a well-known functional analysis result. The same is true for normal operators on Hilbert spaces. Note that, looking at the real and imaginary part, a normal operator corresponds to a pair of commuting selfadjoint operators. For a finite tuple $A_{1}, \ldots, A_{n}$ of self-adjoint operators on a Hilbert space we also have a spectral theorem; see for example [1] or [8]. In fact, there exists a unique compactly supported spectral measure on $\mathbb{R}^{n}$ such that $A_{j}=\int_{\mathbb{R}^{n}} s_{j} d E(\underline{s})$, where $\underline{s}$ denotes a vector in $\mathbb{R}^{n}$ and $s_{j}$ denotes its $j$-th entry.

For a bounded operator on a Krein space the condition being self-adjoint is not rich enough in order to derive some sort of spectral theorem. Assuming in addition definitizability, a spectral theorem could be derived by Heinz Langer; cf. [7]. Here a self-adjoint bounded linear operator $A$ on a Krein space $(\mathcal{K},[.,]$.$) is called definitizable if there exists a so-called definitizing$ polynomial $q(z) \in \mathbb{R}[z] \backslash\{0\}$ such that $[q(A) x, x] \geq 0$ for all $x \in \mathcal{K}$. This theorem became an important starting point for various spectral results. The 
main difference to self-adjoint operators on Hilbert spaces is the appearance of a finite number of critical points, where the spectral projections no longer behave like a measure.

Focusing not on spectral measures but on the corresponding functional calculus the spectral theorem for a definitizable self-adjoint operator on a Krein space was also considered in a somewhat more general form in [6]. The methods used in this work proved to be fruitful enough in order to derive a spectral theorem for a definitizable normal operator in [4], where a normal operator $N$ on a Krein space $\mathcal{K}$ was called definitizable if its real part $A_{1}:=\frac{1}{2}\left(N+N^{+}\right)$and its imaginary part $A_{2}:=\frac{1}{2 \mathrm{i}}\left(N-N^{+}\right)$are both definitizable in the above sense. Here $N^{+}$denotes the adjoint of $N$ with respect to the Krein space inner product [., .]. Using methods from ring theory a spectral theorem for a normal operator satisfying a more general concept of definitizability was proved in [5].

In the present paper we derive a spectral theorem for a finite tuple of pairwise commuting, self-adjoint and definitizable bounded linear operators $\underline{A}=\left(A_{1}, \ldots, A_{n}\right)$ on a Krein space generalizing the ideas from [4]. This will be done in terms of a functional calculus generalizing the functional calculus $\phi \mapsto \int \phi d E$ in the Hilbert space case.

In the preliminary Sect. 2 we will recall some facts about the spectrum of a finite tuple of elements of a Banach algebra. Then we will see that the spectrum of a finite tuple of normal operators on a Hilbert space coincides with the support of the common spectral measure of this tuple of normal operators.

Denoting by $q_{j}(z)$ the definitizing real polynomials for $A_{j}$ we build a Hilbert space $\mathcal{H}$ which is continuously and densely embedded in the given Krein space $\mathcal{K}$. Denoting by $T: \mathcal{H} \rightarrow \mathcal{K}$ the adjoint of the embedding, we have $T T^{+}=\sum_{j=1}^{n} q\left(A_{j}\right)$. Then we use the $*$-homomorphism ${ }^{1} \Theta:\left(T T^{+}\right)^{\prime}(\subseteq$ $\left.L_{b}(\mathcal{K})\right) \rightarrow\left(T^{+} T\right)^{\prime}\left(\subseteq L_{b}(\mathcal{H})\right), C \mapsto(T \times T)^{-1}(C)$, studied in [6], in order to drag $A_{j} \in\left(T T^{+}\right)^{\prime} \subseteq L_{b}(\mathcal{K})$ into $\left(T^{+} T\right)^{\prime} \subseteq L_{b}(\mathcal{H})$. The resulting tuple $\Theta(\underline{A})=\left(\Theta\left(A_{1}\right), \ldots, \Theta\left(A_{n}\right)\right)$ consists of self-adjoint operator on a Hilbert space and therefore has a spectral measure $\Delta \mapsto E(\Delta)$ on the Borel subsets of $\mathbb{R}^{n}$.

The proper family $\mathcal{F}$ of functions suitable for the aimed functional calculus are bounded and measurable functions on the subset $\sigma(\Theta(\underline{A})) \cup$ $\prod_{j=1}^{n} q_{j}^{-1}\{0\}$ of $\mathbb{C}^{n}$. The functions $\phi \in \mathcal{F}$ assume values in $\mathbb{C}$ on $\sigma(\Theta(\underline{A}))$ $\backslash \prod_{j=1}^{n} q_{j}^{-1}\{0\}$ and satisfy $\phi(z) \in \mathbb{C}^{I(z)}$ where $I(z)$ is finite and $\mathbb{C}^{I(z)}$ provided with proper operations constitutes a $*$-algebra. Moreover, a $\phi \in \mathcal{F}$ satisfies a growth regularity condition at all points from $\mathbb{R}^{n} \cap \prod_{j=1}^{n} q_{j}^{-1}\{0\}$ which are not isolated in $\sigma(\Theta(\underline{A})) \cup \prod_{j=1}^{n} q_{j}^{-1}\{0\}$.

\footnotetext{
${ }^{1}$ Given a Krein space $X$ we denote by $L_{b}(X)$ the Banach algebra of all linear and bounded operators on $X$ additionally provided with the Krein space adjoint $B \mapsto B^{+}$.
} 
Any polynomial $s\left(z_{1}, \ldots, z_{n}\right) \in \mathbb{C}\left[z_{1}, \ldots, z_{n}\right]$ can be seen as a function $s_{\mathcal{M}} \in \mathcal{F}$ and any $\phi \in \mathcal{F}$ can be written as

$$
\phi(\underline{z})=s_{\mathcal{M}}(\underline{z})+g(\underline{z}) \cdot\left(\sum_{j=1}^{n}\left(q_{j}\right)_{\mathcal{M}}(\underline{z})\right), \quad \underline{z} \in \sigma(\Theta(\underline{A})) \cup \prod_{j=1}^{n} q_{j}^{-1}\{0\},
$$

for a suitable polynomial $s \in \mathbb{C}\left[z_{1}, \ldots, z_{n}\right]$ and a bounded and measurable function $g: \sigma(\Theta(\underline{A})) \cup \prod_{j=1}^{n} q_{j}^{-1}\{0\} \rightarrow \mathbb{C}$ vanishing on $\prod_{j=1}^{n} q_{j}^{-1}\{0\}$.

We then define $\phi(\underline{A}):=s\left(A_{1}, \ldots, A_{n}\right)+T \int_{\sigma(\Theta(\underline{A}))} g d E T^{+}$, show that this operator does not depend on the actual decomposition (1.1) and that $\phi \mapsto$ $\phi(\underline{A})$ indeed constitutes a $*$-homomorphism. Providing $\mathcal{F}$ with an appropriate norm this $*$-homomorphism is continuous. Finally, we show that for $\underline{A}=$ $\left(A_{1}, A_{2}\right)$ the functional calculus $\phi \mapsto \phi(\underline{A})$ from the present note coincides with the functional calculus derived in [4] for the normal operator $N=$ $A_{1}+\mathrm{i} A_{2}$.

\section{Joint Spectrum of Finite Tuples}

Given a unital and commutative Banach algebra $\mathcal{A}$ with unit $e$ we want to introduce the following notation. For $\underline{a}=\left(a_{j}\right)_{j=1}^{n} \in \mathcal{A}^{n}$ and $\underline{\lambda}=\left(\lambda_{j}\right)_{j=1}^{n} \in \mathbb{C}^{n}$ we define $(\underline{a}-\underline{\lambda}):=\left(a_{j}-\lambda_{j} e\right)_{j=1}^{n}$, for $\underline{b} \in \mathcal{A}^{n}$ we define $\underline{a} \cdot \underline{b}=\sum_{j=1}^{n} a_{j} b_{j}$ and for a mapping $\psi$ defined on $\mathcal{A}$ we set $\psi(\underline{a}):=\left(\psi\left(a_{j}\right)\right)_{j=1}^{n}$.

Denoting by $M$ the maximal ideal space of $\mathcal{A}$ the spectrum of the tuple $\underline{a} \in \mathcal{A}^{n}$ was introduced as

$$
\sigma(\underline{a})=\left\{\phi(\underline{a}) \in \mathbb{C}^{n}: \phi \in M\right\} .
$$

In particular, $\sigma(\underline{a}) \neq \emptyset$. Using well-known results from Gelfand Theory, we see that

$$
\sigma(\underline{a})=\left\{\underline{\lambda} \in \mathbb{C}^{n}: I(\underline{a}-\underline{\lambda}) \neq \mathcal{A}\right\},
$$

where $I(\underline{a}-\underline{\lambda})$ denotes the smallest Ideal containing all entries of $\underline{a}-\underline{\lambda}$. As $\mathcal{A}$ is commutative, $I(\underline{a}-\underline{\lambda})$ coincides with $\left\{\underline{b} \cdot(\underline{a}-\underline{\lambda}): \underline{b} \in \mathcal{A}^{n}\right\}$.

Since an Ideal $I$ satisfies $I \neq \mathcal{A}$ if and only if $e \notin I$, we obtain

$$
\sigma(\underline{a})=\left\{\underline{\lambda} \in \mathbb{C}^{n}:(\underline{a}-\underline{\lambda}) \notin \operatorname{Inv}\left(\mathcal{A}^{n}\right)\right\},
$$

where $\operatorname{Inv}\left(\mathcal{A}^{n}\right)$ is the set of tuples $\underline{c} \in \mathcal{A}^{n}$ such that there exists a tuple $\underline{b} \in \mathcal{A}^{n}$ satisfying $\underline{b} \cdot \underline{c}=e$. Since $\left(c_{j}\right)_{j=1}^{m} \in \operatorname{Inv}\left(\mathcal{A}^{m}\right)$ implies $\underline{c} \in \operatorname{Inv}\left(\mathcal{A}^{n}\right)$ for $m \leq n$, we obtain

$$
\underline{\lambda} \in \sigma(\underline{a}) \Rightarrow\left(\lambda_{j}\right)_{j=1}^{m} \in \sigma\left(\left(a_{j}\right)_{j=1}^{m}\right) .
$$

Definition 2.1. Let $\underline{N}=\left(N_{j}\right)_{j=1}^{n} \in L_{b}(\mathcal{H})^{n}$, where $N_{1}, \ldots, N_{n} \in L_{b}(\mathcal{H})$ are pairwise commuting operators on a Hilbert space $\mathcal{H}$. Then we define $\sigma(\underline{N})$ by (2.1) considering $N_{1}, \ldots, N_{n}$ as elements of the commutative unital algebra $\underline{N}^{\prime \prime}:=\left\{N_{1}, \ldots, N_{n}\right\}^{\prime \prime}$, where $\left\{N_{1}, \ldots, N_{n}\right\}^{\prime \prime}$ denotes the bi-commutant of $\left\{N_{1}, \ldots, N_{n}\right\}$, i.e. the set of all operators on $\mathcal{H}$ commuting with all operators that commute with $N_{1}, \ldots, N_{n}$. 
For an $n$-tuple of normal operators on a Hilbert space a Spectral Theorem is well-known; see for example [8, Theorem 5.21]:

Theorem 2.2. Let $\underline{N}=\left(N_{j}\right)_{j=1}^{n} \in L_{b}(\mathcal{H})^{n}$, where $N_{1}, \ldots, N_{n} \in L_{b}(\mathcal{H})$ are normal and pairwise commuting operators on a Hilbert space $\mathcal{H}$. Then there exists a unique common spectral measure $E$ defined on the Borel-subsets of $\mathbb{C}^{n}$ such that

$$
N_{j}=\int_{\mathbb{C}^{n}} z_{j} d E(\underline{z})
$$

where $z_{j}$ is the $j$-th entry of $\underline{z} \in \mathbb{C}^{n}$. Moreover, an operator $S \in L_{b}(\mathcal{H})$ commutes with all $N_{1}, \ldots, N_{n}$ if and only if $S$ commutes with $E(\Delta)$ for all Borel-subsets $\Delta \subseteq \mathbb{C}^{n}$.

The final assertion in the previous result can be shown with the help of Fuglede's Theorem and the Riesz-Markov Theorem together with the fact that the set of all polynomials in the variables $z_{1}, \ldots, z_{n}, \bar{z}_{1}, \ldots, \bar{z}_{n}$ are dense in $C(\operatorname{supp} E, \mathbb{C})$ with respect to $\|\cdot\|_{\infty}$.

Remark 2.3. The support $\operatorname{supp} E$ of a spectral measure $E$ as in Theorem 2.2 is defined as the set of points $\underline{\lambda} \in \mathbb{C}^{n}$ such that $E(U) \neq 0$ for all measurable neighbourhoods $U$ of $\underline{\lambda}$ in $\mathbb{C}^{n}$. It is easy to check that supp $E$ is a closed subset of $\mathbb{C}^{n}$. By $[8$, Proposition 5.24, (ii)] the support supp $E$ is also bounded, and hence, supp $E$ is compact. For bounded and measurable functions $\phi: \mathbb{C}^{n} \rightarrow \mathbb{C}$ we always have

$$
\int_{\mathbb{C}^{n}} \phi d E=\int_{\mathbb{C}^{n}} \phi \cdot \mathbb{1}_{\operatorname{supp} E} d E .
$$

By [8, Theorem 5.23] the spectral measure $E$ in Theorem 2.2 is supported on $\mathbb{R}^{n}$, i.e. $\operatorname{supp} E \subseteq \mathbb{R}^{n}$, if $N_{1}, \ldots, N_{n}$ are all self-adjoint. Therefore, the integral in (2.4) can be taken over $\mathbb{R}^{n}$ instead of $\mathbb{C}^{n}$ and $E$ can be considered as a spectral measure on the Borel-subsets of $\mathbb{R}^{n}$. $\diamond$

The following result is known. In the absence of a proper reference we also bring its proof.

Theorem 2.4. Let $\underline{N}=\left(N_{j}\right)_{j=1}^{n} \in L_{b}(\mathcal{H})^{n}$, where $N_{1}, \ldots, N_{n} \in L_{b}(\mathcal{H})$ be pairwise commuting normal operators on a Hilbert space $\mathcal{H}$, and denote by $E$ their common spectral measure. Then we have

$$
\sigma(\underline{N})=\operatorname{supp} E,
$$

where $\underline{\lambda} \in \operatorname{supp} E$ if and only if $E(U) \neq 0$ for all measurable neighbourhoods $U$ of $\underline{\lambda}$ in $\mathbb{C}^{n}$.

Proof. If $\underline{\lambda} \in \operatorname{supp} E$, then $E\left(U_{\epsilon}(\underline{\lambda})\right) \neq 0$ for any $\epsilon>0$, where $U_{\epsilon}(\underline{\lambda})$ denotes the open ball of radius $\epsilon$ around $\underline{\lambda}$ in $\mathbb{C}^{n}$ with respect to the Euclidean norm. In particular, there exists an $f_{\epsilon} \in \mathcal{H} \backslash\{0\}$ with $f_{\epsilon}=E\left(U_{\epsilon}(\underline{\lambda})\right) f_{\epsilon}$. We obtain

$$
\begin{aligned}
\left\|\left(N_{j}-\lambda_{j}\right) f_{\epsilon}\right\|^{2} & =\int_{\mathbb{C}^{n}}\left|z_{j}-\lambda_{j}\right|^{2} d\left(E(\underline{z}) f_{\epsilon}, f_{\epsilon}\right)=\int_{U_{\epsilon}(\underline{\lambda})}\left|z_{j}-\lambda_{j}\right|^{2} d\left(E(\underline{z}) f_{\epsilon}, f_{\epsilon}\right) \\
& \leq \epsilon^{2}\left\|f_{\epsilon}\right\|^{2}
\end{aligned}
$$


for all $j \in\{1, \ldots, n\}$. For arbitrary $\underline{B} \in\left(\underline{N}^{\prime \prime}\right)^{n}$ this gives

$$
\left\|\underline{B} \cdot(\underline{N}-\underline{\lambda}) f_{\epsilon}\right\|=\left\|\sum_{j=1}^{n} B_{j}\left(N_{j}-\lambda_{j}\right) f_{\epsilon}\right\| \leq \epsilon \cdot\left(\sum_{j=1}^{n}\left\|B_{j}\right\|\right) \cdot\left\|f_{\epsilon}\right\| .
$$

Taking into account that $\epsilon>0$ can be arbitrarily small, we see that $\underline{B} \cdot(\underline{N}-\underline{\lambda})$ cannot be boundedly invertible. In particular, $\underline{B} \cdot(\underline{N}-\underline{\lambda}) \neq I$ which according to (2.2) yields $\underline{\lambda} \in \sigma(\underline{N})$. where

On the other hand if $\underline{\lambda} \in \mathbb{C}^{n} \backslash \operatorname{supp} E$, then we can define $\underline{B}:=\left(B_{j}\right)_{j=1}^{n}$,

$$
B_{j}:=\int_{\mathbb{C}^{n}} \frac{\mathbb{1}_{\operatorname{supp} E}(\underline{z})}{\|\underline{z}-\underline{\lambda}\|^{2}} \cdot \overline{\left(z_{j}-\underline{\lambda}\right)} d E(\underline{z}),
$$

because the integrand is bounded and measurable, where $\underline{\bar{w}}=\left(\bar{w}_{j}\right)_{j=1}^{n}$. From the final assertion in Theorem 2.2 we infer $\underline{B} \in\left(\underline{N}^{\prime \prime}\right)^{n}$. By

$$
\begin{aligned}
\underline{B} \cdot(\underline{N}-\underline{\lambda}) & =\sum_{j=1}^{n} \int_{\mathbb{C}^{n}} \frac{\mathbb{1}_{\operatorname{supp} E}(\underline{z})}{\|\underline{z}-\underline{\lambda}\|^{2}} \overline{\left(z_{j}-\lambda_{j}\right)} \cdot\left(z_{j}-\lambda_{j}\right) d E(\underline{z}) \\
& =\int_{\mathbb{C}^{n}} \frac{\mathbb{1}_{\operatorname{supp} E}(\underline{z})}{\|\underline{z}-\underline{\lambda}\|^{2}} \cdot \sum_{j=1}^{n}\left|z_{j}-\lambda_{j}\right|^{2} d E(\underline{z}) \\
& =\int_{\mathbb{C}^{n}} \mathbb{1}_{\operatorname{supp} E} d E=I
\end{aligned}
$$

we conclude from (2.2) that $\underline{\lambda} \notin \sigma(\underline{N})$.

The uniqueness assertion in Theorem 2.2 yields the following description of the unique common spectral measure for a shortened tuple $\left(N_{j}\right)_{j=1}^{m}$.

Theorem 2.5. With the notation of Theorem 2.2 let $m \in \mathbb{N}$ with $m \leq n$. The unique common spectral measure from Theorem 2.2 for the tuple $\left(N_{j}\right)_{j=1}^{m}$ is given by

$$
E\left(\pi^{-1}(\Delta)\right)
$$

for all Borel-subsets $\Delta \subseteq \mathbb{C}^{m}$, where $\pi: \mathbb{C}^{n} \rightarrow \mathbb{C}^{m}$ denotes the projection on the first $m$ components.

In particular, the support of the common spectral measure for the tuple $\left(N_{j}\right)_{j=1}^{m}$ coincides with $\pi(\operatorname{supp} E)$.

\section{Multiple Embeddings}

In the present section we consider a Krein space $(\mathcal{K},[.,]$.$) . The following$ straight forward result implicitly appears in many papers; see for example [4]. For a more detailed discussion and for unitarily equivalent spaces see [2].

Lemma 3.1. Let $D: \mathcal{K} \rightarrow \mathcal{K}$ be a bounded and linear operator which is positive, i.e. $[D x, x] \geq 0$ for all $x \in \mathcal{K}$. Then there exists a Hilbert space $\mathcal{H}$ and an injective, bounded and linear mapping $T: \mathcal{H} \rightarrow \mathcal{K}$ such that $t^{2} T T^{+}=D$.

\footnotetext{
${ }^{2}$ Here $T^{+}: \mathcal{K} \rightarrow \mathcal{H}$ denotes the adjoint of $T$ with respect to the Krein space product $[.,$. on $\mathcal{K}$ and the Hilbert space product on $\mathcal{H}$.
} 
Proof. Since $D$ is positive, $\langle.,\rangle:.=[D .,$.$] defines a positive semidefinite inner$ product on $\mathcal{K}$. Factorizing $\mathcal{K}$ by its isotropic part $\mathcal{K}^{\langle 0\rangle}=\{x \in \mathcal{K}:\langle x, y\rangle=$ 0 for all $y \in \mathcal{K}\}$ we obtain the pre-Hilbert space $\mathcal{K} / \mathcal{K}^{\langle 0\rangle}$ provided with the well-defined positive definite inner product $\left\langle x+\mathcal{K}^{\langle 0\rangle}, y+\mathcal{K}^{\langle 0\rangle}\right\rangle:=\langle x, y\rangle$ for $x, y \in \mathcal{K}$. By

$$
\iota:\left\{\begin{aligned}
\mathcal{K} & \rightarrow \mathcal{K} / \mathcal{K}^{\langle 0\rangle}, \\
x & \mapsto x+\mathcal{K}^{\langle 0\rangle},
\end{aligned}\right.
$$

we denote the factor mapping. Define $(\mathcal{H},\langle.,\rangle$.$) to be the Hilbert space com-$ pletion of $\left(\mathcal{K} / \mathcal{K}^{\langle\circ\rangle},\langle.,\rangle.\right)$ and regard $\iota$ as a mapping into $\mathcal{H}$. From

$$
\|\iota x\|^{2}=\langle\iota x, \iota x\rangle=[D x, x]_{\mathcal{K}} \leq\|D\|\|x\|^{2}, \quad x \in \mathcal{K},
$$

we conclude the continuity of $\iota$. Here the norm on the right hand side is induced by an arbitrary Hilbert space inner product on $\mathcal{K}$ which is compatible with [.,.]. It is well-known that Krein space adjoint $T:=\iota^{+}$of $\iota$, satisfying $[T x, y]=\langle x, \iota y\rangle$ for all $x \in \mathcal{H}$ and $y \in \mathcal{K}$, constitutes a linear and bounded operator $T: \mathcal{H} \rightarrow \mathcal{K}$.

By construction $\operatorname{ran} \iota$ is densely contained in $\mathcal{H}$, which implies $\operatorname{ker} T=$ ker $\iota^{+}=(\operatorname{ran} \iota)^{\langle\perp\rangle}=\{0\}$. Hence, $T$ is injective. Moreover, by definition, for $x, y \in \mathcal{K}$ we have

$$
\left[T T^{+} x, y\right]=\left\langle T^{+} x, T^{+} y\right\rangle=\langle\iota x, \iota y\rangle=\langle x, y\rangle=[D x, y] .
$$

Therefore, $T T^{+}=D$.

Remark 3.2. In Lemma 3.1 we have $\mathcal{H}=\{0\}$ and $T=0$ if $D=0 . \diamond$

Definition 3.3. If bounded linear and positive operators $D_{1}, \ldots, D_{m} \in L_{b}(\mathcal{K})$ are given, then we can apply Lemma 3.1, and obtain for each $j=1, \ldots, m$ a Hilbert space $\mathcal{H}_{j}$ and a bounded linear and injective $T_{j}: \mathcal{H}_{j} \rightarrow \mathcal{K}$ such that $T_{j} T_{j}^{+}=D_{j}$.

Since for any non-empty subset $M \subseteq\{1, \ldots, m\}$ the sum $\sum_{j \in M} D_{j}$ also constitutes a positive operator, we even obtain a Hilbert space $\mathcal{H}_{M}$ and a bounded linear and injective $T_{M}: \mathcal{H}_{M} \rightarrow \mathcal{K}$ such that

$$
T_{M} T_{M}^{+}=\sum_{j \in M} D_{j}
$$

Clearly, $\mathcal{H}_{\{j\}}=\mathcal{H}_{j}$ and $T_{\{j\}}=T_{j}$ for $j=1, \ldots, m . \diamond$

Lemma 3.4. If $M_{1}, \ldots, M_{r}$ are non-empty and pairwise disjoint subsets of $\{1, \ldots, m\}$ and if we set $M:=\bigcup_{k=1}^{r} M_{k}$, then

$$
T_{M} T_{M}^{+}=\sum_{k=1}^{r} T_{M_{k}} T_{M_{k}}^{+} .
$$

Moreover, employing the notation from Definition 3.3, for $k=1, \ldots, r$ there exist injective contractions $R_{M_{k} / M}: \mathcal{H}_{M_{k}} \rightarrow \mathcal{H}_{M}$ such that $T_{M_{k}}=T_{M} R_{M_{k} / M}$ and

$$
\sum_{k=1}^{r} R_{M_{k} / M} R_{M_{k} / M}^{*}=I_{\mathcal{H}_{M}} .
$$




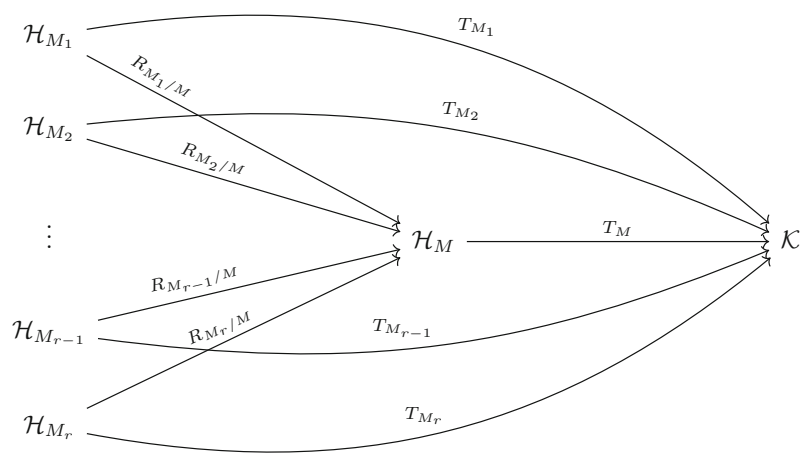

FigURE 1. Setting of Lemma 3.4

Proof. Equation (3.1) clearly follows from $T_{M} T_{M}^{+}=\sum_{j \in M} D_{j}=\sum_{s=1}^{r}$ $\sum_{j \in M_{s}} D_{j}=\sum_{s=1}^{r} T_{M_{s}} T_{M_{s}}^{+}$. For $x \in \mathcal{K}$ we then conclude

$$
\begin{aligned}
\left\|T_{M}^{+} x\right\|_{\mathcal{H}_{M}}^{2} & =\left\langle T_{M}^{+} x, T_{M}^{+} x\right\rangle_{\mathcal{H}_{M}}=\left[T_{M} T_{M}^{+} x, x\right]=\sum_{s=1}^{r}\left[T_{M_{s}} T_{M_{s}}^{+} x, x\right] \\
& =\sum_{s=1}^{r}\left\langle T_{M_{s}}^{+} x, T_{M_{s}}^{+} x\right\rangle_{\mathcal{H}_{M_{s}}}=\sum_{s=1}^{r}\left\|T_{M_{s}}^{+} x\right\|_{\mathcal{H}_{M_{s}}}^{2} \geq\left\|T_{M_{k}}^{+} x\right\|_{\mathcal{H}_{M_{k}}}^{2}
\end{aligned}
$$

for every $k=1, \ldots, r$. This inequality guarantees that

$$
B_{k}:\left\{\begin{aligned}
\operatorname{ran} T_{M}^{+} & \rightarrow \operatorname{ran} T_{M_{k}}^{+}, \\
T_{M}^{+} x & \mapsto T_{M_{k}}^{+} x,
\end{aligned}\right.
$$

is a well-defined, linear and contractive mapping.

Since $T_{M}$ is injective, we have $\left(\operatorname{ran} T_{M}^{+}\right)^{\langle\perp\rangle_{\mathcal{H}_{M}}}=\operatorname{ker} T_{M}=\{0\}$. Hence, $\operatorname{ran} T_{M}^{+}$is dense in $\mathcal{H}_{M}$. The same is true for $\operatorname{ran} T_{M_{k}}^{+}$in $\mathcal{H}_{M_{k}}$. We conclude that $B_{k}$ is densely defined, and hence, its closure $\bar{B}_{k}$ is an everywhere on $\mathcal{H}_{M}$ defined linear contraction with dense range contained in $\mathcal{H}_{M_{k}}$. Thus, its adjoint $R_{M_{k} / M}:=\left(\bar{B}_{k}\right)^{*}$ constitutes an injective linear contractions $R_{M_{k} / M}$ : $\mathcal{H}_{M_{k}} \rightarrow \mathcal{H}_{M}$.

By definition we have $R_{M_{k} / M}^{*} T_{M}^{+}=\bar{B}_{k} T_{M}^{+}=T_{M_{k}}^{+}$, which leads to $T_{M} R_{M_{k} / M}=T_{M_{k}}$. By (3.1) we have

$$
\begin{aligned}
T_{M}\left(I_{\mathcal{H}_{M}}\right) T_{M}^{+} & =T_{M} T_{M}^{+}=\sum_{k=1}^{r} T_{M_{k}} T_{M_{k}}^{+}=\sum_{k=1}^{r} T_{M} R_{M_{k} / M}\left(T_{M} R_{M_{k} / M}\right)^{+} \\
& =T_{M}\left(\sum_{k=1}^{r} R_{M_{k} / M} R_{M_{k} / M}^{*}\right) T_{M}^{+} .
\end{aligned}
$$

Together with the injectivity of $T_{M}$ and the density of $\operatorname{ran} T_{M}^{+}$this yields $I_{\mathcal{H}_{M}}=\sum_{k=1}^{r} R_{M_{k} / M} R_{M_{k} / M}^{*}$ (Fig. 1).

Remark 3.5. If $r=1$ and $M_{1}=M$ in Lemma 3.4, then we realize from the previous proof that $R_{M, M}$ is just the identity mapping on $\mathcal{H}_{M}$. $\diamond$ 
For any Hilbert or Krein space $\mathcal{V}$ and any $B \in L_{b}(\mathcal{V})$ by $B^{\prime}$ we denote the commutant of $\{B\}$, i.e. $B^{\prime}=\left\{C \in L_{b}(\mathcal{V}): C B=B C\right\}$.

Definition 3.6. With the assumptions and notation from Definition 3.3 and from Lemma 3.4 for non-empty $M, N \subseteq\{1, \ldots, m\}$ with $N \subseteq M$ we define

$$
\Theta_{M}: \underbrace{\left(T_{M} T_{M}^{+}\right)^{\prime}}_{\subseteq L_{b}(\mathcal{K})} \rightarrow \underbrace{\left(T_{M}^{+} T_{M}\right)^{\prime}}_{\subseteq} L_{b}\left(\mathcal{H}_{M}\right)
$$

by $^{3} \quad \Theta_{M}(B)=\left(T_{M} \times T_{M}\right)^{-1}(B)=T_{M}^{-1} B T_{M}$ and

$$
\Gamma_{N / M}: \underbrace{\left(R_{N / M} R_{N / M}^{*}\right)^{\prime}}_{\subseteq L_{b}\left(\mathcal{H}_{M}\right)} \rightarrow \underbrace{\left(R_{N / M}^{*} R_{N / M}\right)^{\prime}}_{\subseteq L_{b}\left(\mathcal{H}_{N}\right)}
$$

by $\Gamma_{N / M}(C)=\left(R_{N / M} \times R_{N / M}\right)^{-1}(C)=R_{N / M}^{-1} D R_{N / M} \cdot \diamond$

Remark 3.\%. The mapping $\Theta_{M}\left(\Gamma_{N / M}\right)$ is exactly the mapping $\Theta$ in $[6$, Theorem 5.8] corresponding to the mappings $T=T_{M}\left(T=R_{N / M}\right)$. Therefore, $\Theta_{M}$ and $\Gamma_{N / M}$ constitute $*$-algebra homomorphisms.

The results from [6] dealing with the mapping $\Theta$ could also be shown with the help of the lifting procedure for example discussed in [3, Lemma 2.1]. Probably this lifting procedure allows smoother verifications of the results from [6]. $\diamond$

If $M_{1}, \ldots, M_{r}, M \subseteq\{1, \ldots, m\}$ are as in Lemma 3.4 , then we conclude from (3.1) that

$$
\bigcap_{k=1}^{r}\left(T_{M_{k}} T_{M_{k}}^{+}\right)^{\prime} \subseteq\left(T_{M} T_{M}^{+}\right)^{\prime} .
$$

Therefore, the following result is a consequence of [5, Lemma 2.1] applied to $T_{M_{1}}, \ldots, T_{M_{r}}$.

Proposition 3.8. With the assumptions and notation from Definition 3.3, Lemma 3.4 and Definition 3.6 one has

$$
\Theta_{M}\left(\bigcap_{k=1}^{r}\left(T_{M_{k}} T_{M_{k}}^{+}\right)^{\prime}\right) \subseteq \bigcap_{k=1}^{r}\left(R_{M_{k} / M} R_{M_{k} / M}^{*}\right)^{\prime} \cap\left(T_{M}^{+} T_{M}\right)^{\prime},
$$

where for $s=1, \ldots, r$ and all $B \in \bigcap_{k=1}^{r}\left(T_{M_{k}} T_{M_{k}}^{+}\right)^{\prime}$

$$
\Theta_{M}(B) R_{M_{s} / M} R_{M_{s} / M}^{*}=R_{M_{s} / M} \Theta_{M_{s}}(B) R_{M_{s} / M}^{*}=R_{M_{s} / M} R_{M_{s} / M^{*}}^{*} \Theta_{M}(B),
$$

and

$$
\Theta_{M_{s}}(B)=\Gamma_{M_{s} / M} \circ \Theta_{M}(B)
$$

Lemma 3.9. If $D_{1}, \ldots, D_{m}$ are pairwise commuting operators in Definition 3.3, then for non-empty $N \subseteq M \subseteq\{1, \ldots, m\}$ the operator $R_{N / M} R_{N / M}^{*}$ commutes with $T_{M}^{+} T_{M}$ and $R_{N / M}^{*} R_{N / M}$ commutes with $T_{N}^{+} T_{N}$. Moreover,

$$
\Theta_{M}\left(T_{N} T_{N}^{+}\right)=R_{N / M} R_{N / M}^{*} T_{M}^{+} T_{M}=T_{M}^{+} T_{M} R_{N / M} R_{N / M}^{*}
$$

\footnotetext{
${ }^{3}$ For the middle term the operator $B: \mathcal{K} \rightarrow \mathcal{K}$ has to be identified with its graph, which is a subspace of $\mathcal{K} \times \mathcal{K}$, i.e. a linear relation.
} 
Proof. If $D_{1}, \ldots, D_{m}$ commute pairwise, then $T_{N} T_{N}^{+}=\sum_{j \in N} D_{j}$ commutes with $T_{M} T_{M}^{+}=\sum_{j \in M} D_{j}$. Since

$$
\begin{aligned}
T_{M}\left(T_{M}^{+} T_{M} R_{N / M} R_{N / M}^{*}\right) T_{M}^{+} & =T_{M} T_{M}^{+}\left(T_{M} R_{N / M}\right)\left(R_{N / M}^{*} T_{M}^{+}\right) \\
& =T_{M} T_{M}^{+} T_{N} T_{N}^{+}=T_{N} T_{N}^{+} T_{M} T_{M}^{+} \\
& =T_{M}\left(R_{N / M} R_{N / M}^{*} T_{M}^{+} T_{M}\right) T_{M}^{+},
\end{aligned}
$$

again the injectivity of $T_{M}$ and the density of $\operatorname{ran} T_{M}^{+}$implies that $R_{N / M} R_{N / M}^{*}$ and $T_{M}^{+} T_{M}$ commute, which in turn yields

$$
\begin{aligned}
\left(T_{N}^{+} T_{N}\right)\left(R_{N / M}^{*} R_{N / M}\right) & =\left(R_{N / M}^{*} T_{M}^{+} T_{M} R_{N / M}\right)\left(R_{N / M}^{*} R_{N / M}\right) \\
& =R_{N / M}^{*}\left(T_{M}^{+} T_{M} R_{N / M} R_{N / M}^{*}\right) R_{N / M} \\
& =R_{N / M}^{*} R_{N / M} R_{N / M}^{*} T_{M}^{+} T_{M} R_{N / M} \\
& =\left(R_{N / M}^{*} R_{N / M}\right)\left(T_{N}^{+} T_{N}\right) .
\end{aligned}
$$

Finally, (3.3) follows from

$$
T_{M}^{-1} T_{N} T_{N}^{+} T_{M}=T_{M}^{-1} T_{M} R_{N / M} R_{N / M}^{*} T_{M}^{+} T_{M}=R_{N / M} R_{N / M}^{*} T_{M}^{+} T_{M} .
$$

The following result is a generalization of [5, Corollary 2.3] to $n$ selfadjoint operators.

Corollary 3.10. With the assumptions and notation from Definition 3.3, Lemma 3.4 and Definition 3.6 let $\underline{A}=\left(A_{j}\right)_{j=1}^{n}$, where $A_{1}, \ldots, A_{n} \in L_{b}(\mathcal{K})$ be pairwise commuting self-adjoint operators that are all contained in $\bigcap_{k=1}^{r}\left(T_{M_{k}} T_{M_{k}}^{+}\right)^{\prime}$. Then $\Theta_{M}\left(A_{1}\right), \ldots, \Theta_{M}\left(A_{n}\right) \in L_{b}\left(\mathcal{H}_{M}\right) \quad\left(\Theta_{M_{s}}\left(A_{1}\right), \ldots\right.$, $\left.\Theta_{M_{s}}\left(A_{n}\right) \in L_{b}\left(\mathcal{H}_{M_{s}}\right)\right)$ are pairwise commuting self-adjoint operators on the Hilbert space $\mathcal{H}_{M}\left(\mathcal{H}_{M_{s}}, s=1, \ldots, r\right)$. By $E_{M}\left(E_{M_{s}}\right)$ we denote the common spectral measure of $\Theta_{M}(\underline{A})\left(\Theta_{M_{s}}(\underline{A})\right)$ on the Borel-subsets of $\mathbb{R}^{n}$; see Theorem 2.2 and Remark 2.3.

Then we have $E_{M}(\Delta) \in \bigcap_{k=1}^{r}\left(R_{M_{k} / M} R_{M_{k} / M}^{*}\right)^{\prime} \cap\left(T_{M}^{+} T_{M}\right)^{\prime}$ for all Borelsubsets $\Delta$ of $\mathbb{R}^{n}$ and

$$
\Gamma_{M_{s} / M}\left(E_{M}(\Delta)\right)=E_{M_{s}}(\Delta) \in\left(R_{M_{s} / M}^{*} R_{M_{s} / M}\right)^{\prime} \cap\left(T_{M_{s}}^{+} T_{M_{s}}\right)^{\prime}
$$

for all Borel subsets $\Delta$ of $\mathbb{R}^{n}$. Moreover, $\int h d E_{M} \in \bigcap_{k=1}^{r}\left(R_{M_{k} / M} R_{M_{k} / M}^{*}\right)^{\prime} \cap$ $\left(T_{M}^{+} T_{M}\right)^{\prime}$ and

$$
\Gamma_{M_{s} / M}\left(\int h d E_{M}\right)=\int h d E_{M_{s}} \in\left(R_{M_{s} / M}^{*} R_{M_{s} / M}\right)^{\prime} \cap\left(T_{M_{s}}^{+} T_{M_{s}}\right)^{\prime}
$$

for any bounded and measurable $h: \operatorname{supp} E_{M} \rightarrow \mathbb{C}$ and $s=1, \ldots, r$.

Proof. Since $\Theta_{M}\left(\Theta_{M_{s}}\right)$ is a $*$-homomorphisms, the images of commuting operators commute as well. According to Proposition $3.8 \Theta_{M}\left(A_{j}\right)$ belongs to $\bigcap_{k=1}^{r}\left(R_{M_{k} / M} R_{M_{k} / M}^{*}\right)^{\prime} \cap\left(T_{M}^{+} T_{M}\right)^{\prime}$ for every $j=1, \ldots, n$. By Theorem 2.2 we conclude $E_{M}(\Delta) \in \bigcap_{k=1}^{r}\left(R_{M_{k} / M} R_{M_{k} / M}^{*}\right)^{\prime} \cap\left(T_{M}^{+} T_{M}\right)^{\prime}$ and, in turn, $\int h d E_{M} \in$

\footnotetext{
${ }^{4}$ Note that according to (3.4) we have $\operatorname{supp} E_{M_{s}} \subseteq \operatorname{supp} E_{M}$.
} 
$\bigcap_{k=1}^{r}\left(R_{M_{k} / M} R_{M_{k} / M}^{*}\right)^{\prime} \cap\left(T_{M}^{+} T_{M}\right)^{\prime}$. This also justifies the application of $\Gamma_{M_{k} / M}$ to $E_{M}(\Delta)$ and $\int h d E_{M}$.

The range of $\Theta_{M_{s}}\left(\Gamma_{M_{s} / M}\right)$ is contained in $\left(T_{M_{s}}^{+} T_{M_{s}}\right)^{\prime}\left(\left(R_{M_{s} / M^{*}}^{*} R_{M_{s} / M}\right)^{\prime}\right)$. Again by Theorem 2.2 we obtain $E_{M_{s}}(\Delta), \int h d E_{M_{s}} \in\left(R_{M_{s} / M}^{*} R_{M_{s} / M}\right)^{\prime} \cap$ $\left(T_{M_{s}}^{+} T_{M_{s}}\right)^{\prime}$.

For $C \in\left(R_{M_{s} / M} R_{M_{s} / M}^{*}\right)^{\prime}$ we conclude from [6, Theorem 5.8] that $\Gamma_{M_{s} / M}(C) R_{M_{s} / M}^{*}=R_{M_{s} / M}^{*} C$. For arbitrary $x \in \mathcal{H}_{M}$ and $y \in \mathcal{H}_{M_{s}}$ we therefore have

$$
\begin{aligned}
\left\langle\Gamma_{M_{s} / M}\left(E_{M}(\Delta)\right) R_{M_{s} / M}^{*} x, y\right\rangle_{\mathcal{H}_{M_{s}}} & =\left\langle R_{M_{s} / M}^{*} E_{M}(\Delta) x, y\right\rangle_{\mathcal{H}_{M_{s}}} \\
& =\left\langle E_{M}(\Delta) x, R_{M_{s} / M} y\right\rangle_{\mathcal{H}_{M}}
\end{aligned}
$$

and in turn for and $s \in \mathbb{C}\left[z_{1}, \ldots, z_{n}\right]$

$$
\begin{aligned}
\int_{\mathbb{R}^{n}} s d\left\langle\Gamma_{M_{s} / M}\left(E_{M}(.)\right) R_{M_{s} / M}^{*} x, y\right\rangle_{\mathcal{H}_{M_{s}}}=\int_{\mathbb{R}^{n}} s d\left\langle E_{M}(.) x, R_{M_{s} / M} y\right\rangle_{\mathcal{H}_{M}} \\
=\left\langle s\left(\Theta_{M}(\underline{A})\right) x, R_{M_{s} / M} y\right\rangle_{\mathcal{H}_{M}} \\
=\left\langle R_{M_{s} / M}^{*} s\left(\Theta_{M}(\underline{A})\right) x, y\right\rangle_{\mathcal{H}_{M_{s}}} \\
=\left\langle\Gamma_{M_{s} / M}\left(s\left(\Theta_{M}(\underline{A})\right)\right) R_{M_{s} / M}^{*} x, y\right\rangle_{\mathcal{H}_{M_{s}} .} .
\end{aligned}
$$

From (3.2) and the fact, that $\Gamma_{M_{s} / M}$ is a $*$-homomorphism, we conclude $\Gamma_{M_{s} / M}\left(s\left(\Theta_{M}(\underline{A})\right)\right)=s\left(\Theta_{M_{s}}(\underline{A})\right)$. Therefore,

$$
\begin{aligned}
\int_{\mathbb{R}^{n}} s d\left\langle\Gamma_{M_{s} / M}\left(E_{M}(.)\right) R_{M_{s} / M}^{*} x, y\right\rangle_{\mathcal{H}_{M_{s}}} & =\left\langle s\left(\Theta_{M_{s}}(\underline{A})\right) R_{M_{s} / M}^{*} x, y\right\rangle_{\mathcal{H}_{M_{s}}} \\
& =\int_{\mathbb{R}^{n}} s d\left\langle E_{M_{s}}(.) R_{M_{s} / M}^{*} x, y\right\rangle_{\mathcal{H}_{M_{s}}} .
\end{aligned}
$$

Since supp $E_{M}$ is a compact subset of $\mathbb{R}^{n}, \mathbb{C}\left[z_{1}, \ldots, z_{n}\right]$ is densely contained in $C\left(\operatorname{supp} E_{M}, \mathbb{C}\right)$. The uniqueness assertion in the Riesz-Markov Theorem implies

$$
\left\langle\Gamma_{M_{s} / M}\left(E_{M}(\Delta)\right) R_{M_{s} / M}^{*} x, y\right\rangle_{\mathcal{H}_{M_{s}}}=\left\langle E_{M_{s}}(\Delta) R_{M_{s} / M}^{*} x, y\right\rangle_{\mathcal{H}_{M_{s}}}
$$

and all Borel-subsets $\Delta$ of $\mathbb{R}^{n}$. Since $x \in \mathcal{H}_{M}$ was arbitrary, the density of $\operatorname{ran} R_{M_{s} / M}^{*}$ yields $\left\langle\Gamma_{M_{s} / M}\left(E_{M}(\Delta)\right) z, y\right\rangle_{\mathcal{H}_{M_{s}}}=\left\langle E_{M_{s}}(\Delta) z, y\right\rangle_{\mathcal{H}_{M_{s}}}$ for all $y, z \in \mathcal{H}_{M_{s}}$. Consequently, $\Gamma_{M_{s} / M}\left(E_{M}(\Delta)\right)=E_{M_{s}}(\Delta)$.

From the already proven fact that $E_{M_{s}}(\Delta) R_{M_{s} / M}^{*}=\Gamma_{M_{s} / M}\left(E_{M}(\Delta)\right)$ $R_{M_{s} / M}^{*}=R_{M_{s} / M}^{*} E_{M}(\Delta)$ we obtain for bounded and measurable $h: \operatorname{supp} E_{M} \rightarrow$ $\mathbb{C}$ and $x \in \mathcal{H}_{M}, y \in \mathcal{H}_{M_{s}}$

$$
\begin{array}{r}
\left\langle\Gamma_{M_{s} / M}\left(\int h d E_{M}\right) R_{M_{s} / M}^{*} x, y\right\rangle_{\mathcal{H}_{M_{s}}}=\left\langle R_{M_{s} / M}^{*}\left(\int h d E_{M}\right) x, y\right\rangle_{\mathcal{H}_{M_{s}}} \\
=\left\langle\left(\int h d E_{M}\right) x, R_{M_{s} / M} y\right\rangle_{\mathcal{H}_{M}}=\int h d\left\langle E_{M}(.) x, R_{M_{s} / M} y\right\rangle_{\mathcal{H}_{M}}
\end{array}
$$




$$
=\int h d\left\langle E_{M_{s}}(.) R_{M_{s} / M}^{*} x, y\right\rangle_{\mathcal{H}_{M_{s}}}=\left\langle\left(\int h d E_{M_{s}}\right) R_{M_{s} / M}^{*} x, y\right\rangle_{\mathcal{H}_{M_{s}}} .
$$

Again the density of $\operatorname{ran} R_{M_{s} / M}^{*}$ yields the desired equation (3.5).

Finally in this section, we will introduce mappings of the same kind as considered in [6, Lemma 5.11]. With the assumptions and notation from Definition 3.3 and from Lemma 3.4 for non-empty $M \subseteq\{1, \ldots, m\}$ we define

$$
\Xi_{M}: \begin{cases}L_{b}\left(\mathcal{H}_{M}\right) & \rightarrow L_{b}(\mathcal{K}), \\ C & \mapsto T_{M} C T_{M}^{+} .\end{cases}
$$

For non-empty $N \subseteq M \subseteq\{1, \ldots, m\}$ we define accordingly

$$
\Lambda_{N / M}:\left\{\begin{aligned}
L_{b}\left(\mathcal{H}_{N}\right) & \rightarrow L_{b}\left(\mathcal{H}_{M}\right) \\
C & \mapsto R_{N / M} C R_{N / M}^{*}
\end{aligned}\right.
$$

By Lemma 3.4,

$$
\begin{aligned}
\Xi_{N}(C)= & T_{N} C T_{N}^{*}=T_{M} R_{N / M} C R_{N / M}^{*} T_{M}^{*} \\
= & \Xi_{M} \circ \Lambda_{N / M}(C) \\
& \quad \text { for } C \in L_{b}\left(\mathcal{H}_{N}\right) .
\end{aligned}
$$

According to $\left[6\right.$, Lemma 5.11] for $C \in\left(R_{N / M} R_{N / M}^{*}\right)^{\prime}$ we have

$$
\Lambda_{N / M} \circ \Gamma_{N / M}(C)=R_{N / M} R_{N / M}^{*} C .
$$

Hence, using Corollary 3.10 and its notation together with (3.7) and (3.8) we obtain

$$
\begin{aligned}
\Xi_{N}\left(\int h d E_{N}\right) & =\Xi_{N} \circ \Gamma_{N / M}\left(\int h d E_{M}\right)=\Xi_{M} \circ \Lambda_{N / M} \circ \Gamma_{N / M}\left(\int h d E_{M}\right) \\
& =\Xi_{M}\left(R_{N / M} R_{N / M}^{*} \int h d E_{M}\right) .
\end{aligned}
$$

\section{Tuples of Definitizable Operators on a Krein Space}

In the present section we start with a finite tuple $\underline{A}=\left(A_{j}\right)_{j=1}^{n} \in L_{b}(\mathcal{K})^{n}$ of pairwise commuting, bounded and self-adjoint operators on a Krein space $\mathcal{K}$. We also assume that $A_{1}, \ldots, A_{n}$ are definitizable, i.e. $q_{j}\left(A_{j}\right)$ is positive for some non-zero polynomial $q_{j} \in \mathbb{R}[\zeta]$ for all $j=1, \ldots, n$. Such polynomials $q_{j}$ are called definitizing polynomials for $A_{j}$; see [7].

Employing Definition 3.3 with $D_{1}:=q_{1}\left(A_{1}\right), \ldots, D_{n}:=q_{n}\left(A_{n}\right)$, we obtain Hilbert space $\mathcal{H}_{j}$ and an injective, bounded linear $T_{j}: \mathcal{H}_{j} \rightarrow \mathcal{K}$ such that

$$
T_{j} T_{j}^{+}=q_{j}\left(A_{j}\right) \text { for all } j=1, \ldots, n .
$$

More generally, for any non-empty subset $M \subseteq\{1, \ldots, n\}$ we obtain a Hilbert space $\mathcal{H}_{M}$ and an injective, bounded linear $T_{M}: \mathcal{H}_{M} \rightarrow \mathcal{K}$ such that $T_{M} T_{M}^{+}=$ $\sum_{j \in M} q_{j}\left(A_{j}\right)$.

The fact that $A_{1}, \ldots, A_{n}$ commute pairwise implies that the operators $T_{j} T_{j}^{+}=q_{j}\left(A_{j}\right), j=1, \ldots, n$, commute pairwise and that $A_{1}, \ldots, A_{n} \in$ 
$\left(T_{M} T_{M}^{+}\right)^{\prime}=\left(\sum_{j \in M} q_{j}\left(A_{j}\right)\right)^{\prime}$ for all $\emptyset \neq M \subseteq\{1, \ldots, n\}$. Thus, we can apply all the results from the previous section.

Lemma 4.1. With the assumptions and notation from the present section, with $R_{N / M}: \mathcal{H}_{N} \rightarrow \mathcal{H}_{M}$ as defined in Lemma 3.4 for $\emptyset \neq N \subseteq M \subseteq$ $\{1, \ldots, n\}$ and with the notion from Definition 3.6 we have

$$
\sum_{j \in N} q_{j}\left(\Theta_{M}\left(A_{j}\right)\right)=R_{N / M} R_{N / M}^{*} \sum_{j \in M} q_{j}\left(\Theta_{M}\left(A_{j}\right)\right),
$$

where $R_{N / M} R_{N / M}^{*}$ commutes with $\sum_{j \in M} q_{j}\left(\Theta_{M}\left(A_{j}\right)\right)$.

Proof. From (3.3) together with Remark 3.5 and the fact the $\Theta_{M}$ is a homomorphism we infer

$$
\begin{aligned}
T_{M}^{+} T_{M} & =\Theta_{M}\left(T_{M} T_{M}^{+}\right)=\Theta_{M}\left(\sum_{j \in M} q_{j}\left(A_{j}\right)\right) \\
& =\sum_{j \in M} \Theta_{M}\left(q_{j}\left(A_{j}\right)\right)=\sum_{j \in M} q_{j}\left(\Theta_{M}\left(A_{j}\right)\right) .
\end{aligned}
$$

By Lemma 3.9 the operator $R_{N / M} R_{N / M}^{*}$ commutes with this expression. Finally we again conclude from (3.3)

$$
\begin{aligned}
\sum_{j \in N} q_{j}\left(\Theta_{M}\left(A_{j}\right)\right) & =\Theta_{M}\left(\sum_{j \in N} q_{j}\left(A_{j}\right)\right)=\Theta_{M}\left(T_{N} T_{N}^{+}\right) \\
& =R_{N / M} R_{N / M}^{*} T_{M}^{+} T_{M}=R_{N / M} R_{N / M}^{*} \sum_{j \in M} q_{j}\left(\Theta_{M}\left(A_{j}\right)\right) .
\end{aligned}
$$

Proposition 4.2. With the assumptions and notation from the present section and with the notion from Definition 3.6 for $\emptyset \neq N \subseteq M \subseteq\{1, \ldots, n\}$ let $E_{M}$ denote the common spectral measure of $\Theta_{M}(\underline{A})$ on the Borel-subsets of $\mathbb{R}^{n}$; see Theorem 2.2 and Remark 2.3. Then we have

$\left\{\underline{\lambda} \in \mathbb{R}^{n}:\left|\sum_{j \in N} q_{j}\left(\lambda_{j}\right)\right|>\left\|R_{N / M} R_{N / M}^{*}\right\| \cdot\left|\sum_{j \in M} q_{j}\left(\lambda_{j}\right)\right|\right\} \subseteq \mathbb{C}^{n} \backslash \sigma\left(\Theta_{M}(\underline{A})\right)$.

In particular, the zeros of $\underline{\lambda} \mapsto \sum_{j \in M} q_{j}\left(\lambda_{j}\right)$ are contained in

$$
\left(\mathbb{C}^{n} \backslash \sigma\left(\Theta_{M}(\underline{A})\right)\right) \cup\left\{\underline{\lambda} \in \mathbb{R}^{n}: q_{j}\left(\lambda_{j}\right)=0 \text { for all } j \in\{1, \ldots, n\}\right\} .
$$

Proof. For $m \in \mathbb{N}$ we set

$$
\Delta_{m}:=\left\{\underline{\lambda} \in \mathbb{R}^{n}:\left|\sum_{j \in N} q_{j}\left(\lambda_{j}\right)\right|^{2}>\frac{1}{m}+\left\|R_{N / M} R_{N / M}^{*}\right\|^{2} \cdot\left|\sum_{j \in M} q_{j}\left(\lambda_{j}\right)\right|^{2}\right\} .
$$

If $x \in \operatorname{ran} E_{M}\left(\Delta_{m}\right)$, then we have

$$
\left\|\sum_{j \in N} q_{j}\left(\Theta_{M}\left(A_{j}\right)\right) x\right\|^{2}=\left\|\sum_{j \in N} q_{j}\left(\Theta_{M}\left(A_{j}\right)\right) E\left(\Delta_{m}\right) x\right\|^{2}
$$




$$
\begin{aligned}
& =\int_{\Delta_{m}}\left|\sum_{j \in N} q_{j}\left(z_{j}\right)\right|^{2} d\left(E_{M}(\underline{z}) x, x\right) \\
& \geq \int_{\Delta_{m}} \frac{1}{m} d\left(E_{M}(\underline{z}) x, x\right) \\
& \quad+\left\|R_{N / M} R_{N / M}^{*}\right\|^{2} \int_{\Delta_{m}}\left|\sum_{j \in M} q_{j}\left(z_{j}\right)\right|^{2} d\left(E_{M}(\underline{z}) x, x\right) \\
& \geq \frac{1}{m}\|x\|^{2}+\|\underbrace{R_{N / M} R_{N / M}^{*} \sum_{j \in M} q_{j}\left(\Theta_{M}\left(A_{j}\right)\right) x}_{=\sum_{j \in N} q_{j}\left(\Theta_{M}\left(A_{j}\right)\right) x}\|^{2} .
\end{aligned}
$$

This inequality can only hold true for $x=0$. Hence, $E_{M}\left(\Delta_{m}\right)=0$. By Theorem 2.4 the fact that $\Delta_{m}$ is open yields

$$
\Delta_{m} \subseteq \mathbb{C}^{n} \backslash \operatorname{supp} E_{M}=\mathbb{C}^{n} \backslash \sigma\left(\Theta_{M}(\underline{A})\right) .
$$

Taking the union over all $m \in \mathbb{N}$ we obtain

$$
\begin{array}{r}
\left\{\underline{\lambda} \in \mathbb{R}^{n}:\left|\sum_{j \in N} q_{j}\left(\lambda_{j}\right)\right|>\left\|R_{N / M} R_{N / M}^{*}\right\| \cdot\left|\sum_{j \in M} q_{j}\left(\lambda_{j}\right)\right|\right\} \\
=\bigcup_{m \in \mathbb{N}} \Delta_{m} \subseteq \mathbb{C}^{n} \backslash \sigma\left(\Theta_{M}(\underline{A})\right) .
\end{array}
$$

If $\sum_{j \in M} q_{j}\left(z_{j}\right)=0$ and $\underline{z} \notin\left\{\underline{\lambda} \in \mathbb{R}^{n}: q_{j}\left(\lambda_{j}\right)=0\right.$ for all $\left.j \in\{1, \ldots, n\}\right\}$ then $\left|q_{k}\left(z_{k}\right)\right|>0=\left\|R_{\{k\} / M} R_{\{k\} / M}^{*}\right\| \cdot\left|\sum_{j \in M} q_{j}\left(z_{j}\right)\right|$ for some $k \in\{1, \ldots, n\}$. From the already shown applied to $N=\{k\}$ we conclude $\underline{z} \notin \sigma\left(\Theta_{M}(\underline{A})\right)$.

Corollary 4.3. With the notation and assumptions from Proposition 4.2 and $\Delta:=\left\{\underline{\lambda} \in \mathbb{R}^{n}: q_{k}\left(\lambda_{k}\right) \neq 0\right.$ for some $\left.k \in\{1, \ldots, n\}\right\}$ we have

$$
R_{N / M} R_{N / M}^{*} E_{M}(\Delta)=\int_{\Delta} \frac{\sum_{j \in N} q_{j}\left(z_{j}\right)}{\sum_{j \in M} q_{j}\left(z_{j}\right)} d E_{M}(\underline{z}) .
$$

Proof. By Proposition 4.2 the zeros of $\operatorname{supp} E_{M} \ni \underline{\lambda} \mapsto \sum_{j \in M} q_{j}\left(\lambda_{j}\right)$ are contained in $\mathbb{R}^{n} \backslash \Delta$ and we have

$$
\left|\sum_{j \in N} q_{j}\left(\lambda_{j}\right)\right| \leq\left\|R_{N / M} R_{N / M}^{*}\right\| \cdot\left|\sum_{j \in M} q_{j}\left(\lambda_{j}\right)\right|
$$

for every $\underline{\lambda} \in \operatorname{supp} E_{M}$. Hence, the integrand is bounded on $\Delta \cap \operatorname{supp} E_{M}$ and consequently the integral in (4.3) does exist.

For $0 \neq x \in \mathcal{U}:=\operatorname{ran} E_{M}(\Delta)$ we have

$$
\left\|\int \sum_{j \in M} \overline{q_{j}\left(z_{j}\right)} d E_{M}(\underline{z}) x\right\|^{2}=\left\|\int \sum_{j \in M} \overline{q_{j}\left(z_{j}\right)} d E_{M}(\underline{z}) E_{M}(\Delta) x\right\|^{2}
$$




$$
=\int_{\Delta} \underbrace{\left|\sum_{j \in M} q_{j}\left(z_{j}\right)\right|^{2}}_{>0 \text { on } \Delta} d\left(E_{M}(\underline{z}) x, x\right)>0
$$

and for $x \in \mathcal{U}^{\perp}=\operatorname{ran} E_{M}\left(\mathbb{R}^{n} \backslash \Delta\right)$ we have

$$
\left\|\int \sum_{j \in M} \overline{q_{j}\left(z_{j}\right)} d E_{M}(\underline{z}) x\right\|^{2}=\int_{\mathbb{R}^{n} \backslash \Delta} \underbrace{\left|\sum_{j \in M} q_{j}\left(z_{j}\right)\right|^{2}}_{=0 \text { on } \mathbb{R}^{n} \backslash \Delta} d\left(E_{M}(\underline{z}) x, x\right)=0 .
$$

Therefore, $\mathcal{U}^{\perp}=\operatorname{ker}\left(\int \sum_{j \in M} q_{j}\left(z_{j}\right) d E_{M}(\underline{z})\right)^{*}$. Consequently, the range of $\int \sum_{j \in M} q_{j}\left(z_{j}\right) d E_{M}(\underline{z})$ is densely contained in $\mathcal{U}$. Every $x$ from in this dense subspace can be written as $x=\int \sum_{j \in M} q_{j}\left(z_{j}\right) d E_{M}(\underline{z}) y$ for some $y \in \mathcal{U}$. We obtain from Lemma 4.1

$$
\begin{aligned}
\int_{\Delta} \frac{\sum_{j \in N} q_{j}\left(z_{j}\right)}{\sum_{j \in M} q_{j}\left(z_{j}\right)} d E_{M}(\underline{z}) x & =\int_{\Delta} \sum_{j \in N} q_{j}\left(z_{j}\right) d E_{M}(\underline{z}) y=\sum_{j \in N} q_{j}\left(\Theta_{M}\left(A_{j}\right)\right) y \\
& =R_{N / M} R_{N / M}^{*} \sum_{j \in M} q_{j}\left(\Theta_{M}\left(A_{j}\right)\right) y=R_{N / M} R_{N / M}^{*} x .
\end{aligned}
$$

The density of the space of the considered $x$ in $\mathcal{U}$ finally yields (4.3).

Remark 4.4. In the present section we did not exclude the possibility that $q_{j}\left(A_{j}\right)=0$ for some $j=1, \ldots, n$. In this case we have $\mathcal{H}_{j}=\{0\}$ and $T_{j}=0$. Interpreting the appearing operators involving $\mathcal{H}_{j}$ as zero and their spectrum as the emptyset all results in the present section remain true. $\diamond$

\section{Special Function Classes}

For $n \in \mathbb{N}$ a subset $I \subseteq \mathbb{Z}^{n}$ is called an interval if $\alpha, \beta \in I$ and $\gamma \in \mathbb{Z}^{n}$ with $\alpha_{j} \leq \gamma_{j} \leq \beta_{j}$ for all $j=1, \ldots, n$ implies $\gamma \in I$.

Example 5.1. Given $\alpha, \beta \in \mathbb{Z}^{n}$ the following subsets

$$
\begin{aligned}
& {[\alpha, \beta):=\left\{\gamma \in \mathbb{Z}^{n}: \alpha_{j} \leq \gamma_{j}<\beta_{j} \text { for all } j=1, \ldots, n\right\},} \\
& {[\alpha, \beta]:=\left\{\gamma \in \mathbb{Z}^{n}: \alpha_{j} \leq \gamma_{j} \leq \beta_{j} \text { for all } j=1, \ldots, n\right\},} \\
& {[\alpha, \beta]:=\left\{\gamma \in[\alpha, \beta]: \#\left\{j \in\{1, \ldots, n\}: \gamma_{j}<\beta_{j}\right\} \geq n-1\right\},}
\end{aligned}
$$

of $\mathbb{Z}^{n}$ are intervals. If $\alpha_{j}<\beta_{j}$ for all $j=1, \ldots, n$, then

$$
[\alpha, \beta\rceil=[\alpha, \beta) \cup\left\{\beta_{1} \cdot e_{1}, \ldots, \beta_{n} \cdot e_{n}\right\},
$$

where $e_{j} \in \mathbb{Z}^{n}$ has 1 at position $j$ and zero elsewhere. $\diamond$

Definition 5.2. For $n \in \mathbb{N}$ and an interval $I \subseteq\left(\mathbb{N}_{0}\right)^{n}$ with $(0, \ldots, 0) \in I$ we provide $\mathbb{C}^{I}$ with componentwise addition, scalar multiplication, componentwise complex conjugation $\bar{a}:=\left(\bar{a}_{j}\right)_{j \in I}$ for $a=\left(a_{j}\right)_{j \in I}$ and a multiplication . : $\mathbb{C}^{I} \times \mathbb{C}^{I} \rightarrow \mathbb{C}^{I}$ defined by 


$$
a \cdot b:=\left(\sum_{\beta+\gamma=\alpha} a_{\beta} b_{\gamma}\right)_{\alpha \in I}
$$

for $a, b \in \mathbb{C}^{I}$.

Moreover, for intervals $I \subset J \subseteq\left(\mathbb{N}_{0}\right)^{n}$ let $\pi_{J, I}: \mathbb{C}^{J} \rightarrow \mathbb{C}^{I}$ denote the projection $\pi_{J, I}\left(\left(a_{j}\right)_{j \in J}\right)=\left(a_{j}\right)_{j \in I} . \diamond$

Remark 5.3. Given an interval $I \subseteq\left(\mathbb{N}_{0}\right)^{n}$ with $(0, \ldots, 0) \in I$ the set $\mathbb{C}^{I}$ endowed with the operations introduced in Definition 5.2 forms a unital and commutative $*$-algebra. Its unit is given by $e=\left(e_{\alpha}\right)_{\alpha \in I}$ with $e_{(0, \ldots, 0)}=1$ and $e_{\alpha}=0$ for $\alpha \neq 0$. Moreover, it is easy to check that an element $a \in \mathbb{C}^{I}$ has a multiplicative inverse in $\mathbb{C}^{I}$ if and only if $a_{(0, \ldots, 0)} \neq 0 . \diamond$

Definition 5.4. For a polynomial $p \in \mathbb{C}[\zeta] \backslash\{0\}$ we denote its zero set by

$$
Z_{p}:=\{\zeta \in \mathbb{C}: p(\zeta)=0\}
$$

and we define the function

$$
\mathfrak{d}_{p}:\left\{\begin{array}{l}
\mathbb{C} \rightarrow \mathbb{N}_{0}, \\
\zeta \mapsto \min \left\{j \in \mathbb{N}_{0}: p^{(j)}(\zeta) \neq 0\right\}
\end{array} .\right.
$$

For a fixed tuple $q=\left(q_{1}, \ldots, q_{n}\right) \in(\mathbb{C}[\zeta] \backslash\{0\})^{n}$ of polynomials and $\underline{z} \in \mathbb{C}^{n}$ we employ the notation

$$
\mathfrak{d}_{\underline{q}}(\underline{z}):=\left(\mathfrak{d}_{q_{j}}\left(z_{j}\right)\right)_{j=1}^{n} \in\left(\mathbb{N}_{0}\right)^{n},
$$

and define the following subsets of $\mathbb{C}^{n}$

$$
Z_{\underline{q}}:=\prod_{j=1}^{n} Z_{q_{j}}, \quad Z_{\underline{q}}^{\mathbb{R}}:=Z_{\underline{q}} \cap \mathbb{R}^{n}, \quad Z_{\underline{q}}^{\mathrm{i}}:=Z_{\underline{q}} \backslash \mathbb{R}^{n} .
$$

Finally, we define $I: \mathbb{C}^{n} \rightarrow \mathcal{P}\left(\left(\mathbb{N}_{0}\right)^{n}\right)$ by

$$
I(\underline{z})= \begin{cases}\{(0, \ldots, 0)\}, & \text { if } \underline{z} \notin Z_{\underline{q}}, \\ {\left[0, \mathfrak{d}_{\underline{q}}(\underline{z})\right],} & \text { if } \underline{z} \in Z_{\underline{q}}^{\mathbb{R}}, \\ {\left[0, \mathfrak{d}_{\underline{q}}(\underline{z})\right),} & \text { if } \underline{z} \in Z_{\underline{q}}^{\mathrm{i}} .\end{cases}
$$

In the following we assume $\underline{A}=\left(A_{i}\right)_{i=1}^{n}$ to be a tuple in $L_{b}(\mathcal{K})$, where $A_{1}, \ldots, A_{n} \in L_{b}(\mathcal{K})$ are pairwise commuting, bounded and self-adjoint operators on a Krein space $\mathcal{K}$, which are definitizable. Moreover, let $q_{j} \in \mathbb{R}[\zeta] \backslash\{0\}$ be fixed definitizing polynomials for $A_{j}$, i.e. $q_{j}\left(A_{j}\right)$ is positive for $j=1, \ldots, n$. We use the notation from the previous section. For short we will write $\mathcal{H}$ for $\mathcal{H}_{\{1, \ldots, n\}}, T$ for $T_{\{1, \ldots, n\}}, \Theta$ for $\Theta_{\{1, \ldots, n\}}$ and $R_{j}$ for $R_{\{j\} /\{1, \ldots, n\}}$.

Definition 5.5. With $\underline{q}=\left(q_{1}, \ldots, q_{n}\right)$ let $\mathcal{M}$ be the set of all functions

$$
\phi: \underbrace{\sigma(\Theta(\underline{A})) \cup Z_{q}}_{\subseteq \mathbb{C}^{n}} \rightarrow \bigcup_{M \subseteq\left(\mathbb{N}_{0}\right)^{n}} \mathbb{C}^{M}
$$

with $\phi(\underline{z}) \in \mathbb{C}^{I(\underline{z})}$ for all $\underline{z} \in \sigma(\Theta(\underline{A})) \cup Z_{\underline{q}}$. 
We endow $\mathcal{M}$ with pointwise scalar multiplication, addition and multiplication, where the operations on $\mathbb{C}^{I(\underline{z})}$ are as in ${ }^{5}$ Definition 5.2. For $\phi \in \mathcal{M}$ also define

$$
\phi^{\#}(\underline{z})=\overline{\phi(\underline{\bar{z}})} \text { for } \underline{z} \in \sigma(\Theta(\underline{A})) \cup Z_{\underline{q}} .
$$

Since $q_{j}$ has real coefficients for $j=1, \ldots, n$, we have $I(\underline{\bar{z}})=I(\underline{z})$. Hence, $\phi^{\#} \in \mathcal{M}$ and $.^{\#}: \mathcal{M} \rightarrow \mathcal{M}$ is a conjugate linear involution.

Remark 5.6. Using Remark 5.3 it is easy to check that $\mathcal{M}$ constitutes a commutative $*$-algebra. $\diamond$

For $\underline{z} \in \mathbb{C}^{n}$ and $\alpha \in\left(\mathbb{N}_{0}\right)^{n}$ we shall employ the following handy notion

$$
\underline{z}^{\alpha}=\prod_{j=1}^{n} z_{j}^{\alpha_{j}}, \quad \alpha !=\prod_{j=1}^{n} \alpha_{j} !, \quad|\alpha|=\sum_{j=1}^{n} \alpha_{j} .
$$

Definition 5.7. Let $f: \operatorname{dom} f \rightarrow \mathbb{C}$ be a function with

$$
\sigma(\Theta(\underline{A})) \cup Z_{p} \subseteq \operatorname{dom} f \subseteq \mathbb{C}^{n},
$$

such that $f$ is sufficiently smooth-more exactly, at least

$$
\max _{\underline{w} \in Z_{\underline{q}}^{\mathbb{R}}}\left|\mathfrak{d}_{\underline{q}}(\underline{w})\right|-n+1
$$

times continuously differentiable - on an open neighborhood of $Z_{\underline{q}}^{\mathbb{R}}$ as subset of $\mathbb{R}^{n}$, and such that $f$ is holomorphic on an open neighborhood of $Z_{\underline{q}}^{\mathrm{i}}$ as subset of $\mathbb{C}^{n}$. Then we define $f_{\mathcal{M}} \in \mathcal{M}$ by

$$
f_{\mathcal{M}}(\underline{z}):= \begin{cases}f(\underline{z}) & \text { if } z \in \sigma(\Theta(\underline{A})) \backslash Z_{\underline{q}}^{\mathbb{R}}, \\ \left(\frac{1}{\alpha !} D^{\alpha} f(\underline{z})\right)_{\alpha \in I(\underline{z})}, & \text { if } z \in Z_{\underline{q} .}\end{cases}
$$

For $\underline{z} \in Z_{\underline{q}}^{\mathbb{R}}$ the higher derivative $D^{\alpha}$ should be understood in the sense of real derivation and for $\underline{z} \in Z_{\underline{q}}^{\mathrm{i}}$ in the sense of complex derivation. $\diamond$

Remark 5.8. Let $f, g$ be functions which satisfy the conditions of Definition 5.7. For $\underline{z} \in Z_{\underline{q}}$ and $\alpha \in I(\underline{z})$ the Leibniz rule yields

$$
\begin{aligned}
\left((f g)_{\mathcal{M}}(\underline{z})\right)_{\alpha} & =\frac{1}{\alpha !} D^{\alpha}(f g)(\underline{z})=\frac{1}{\alpha !} \sum_{\beta+\gamma=\alpha} \frac{\alpha !}{\beta ! \gamma !} D^{\beta} f(\underline{z}) D^{\gamma} g(\underline{z}) \\
& =\sum_{\beta+\gamma=\alpha} \frac{1}{\beta !} D^{\beta} f(\underline{z}) \frac{1}{\gamma !} D^{\gamma} g(\underline{z})=\left(f_{\mathcal{M}}(\underline{z}) \cdot g_{\mathcal{M}}(\underline{z})\right)_{\alpha} .
\end{aligned}
$$

Therefore, $(f g)_{\mathcal{M}}(\underline{z})=f_{\mathcal{M}}(\underline{z}) \cdot g_{\mathcal{M}}(\underline{z})$. Consequently, $(f g)_{\mathcal{M}}=f_{\mathcal{M}} \cdot g_{\mathcal{M}}$. Moreover, it is easy to check that for $\lambda, \mu \in \mathbb{C}$

$$
(\lambda f+\mu g)_{\mathcal{M}}=\lambda f_{\mathcal{M}}+\mu g_{\mathcal{M}} .
$$

\footnotetext{
${ }^{5}$ Recall from $(5.2)$ that $I(\underline{z})$ constitutes an interval for all $\underline{z}$.
} 
Furthermore, we define the function $f^{\#}$ by $f^{\#}(\underline{z})=\overline{f(\bar{z})}$ for $\underline{z} \in \operatorname{dom} f$ and immediately convince ourselves that also $f^{\#}$ satisfies the conditions of Definition 5.7 and that

$$
\left(f^{\#}\right)_{\mathcal{M}}=\left(f_{\mathcal{M}}\right)^{\#} .
$$

Example 5.9. Let $j \in\{1, \ldots, n\}$ be fixed and $q_{j}$ be a real definitizing polynomial of $A_{j}$. Then we can regard $q_{j}$ also as an element of $\mathbb{C}\left[z_{1}, \ldots, z_{n}\right]$ by setting $q_{j}(\underline{z})=q_{j}\left(z_{j}\right)$ for $\underline{z} \in \mathbb{C}^{n}$. Clearly, $q_{j}: \mathbb{C}^{n} \rightarrow \mathbb{C}$ satisfies all conditions of Definition 5.7. Since $q_{j}(\underline{z})$ is constant in every direction $z_{k}$ for $k \neq j$, every derivative in these directions vanishes. For $z \in Z_{\underline{q}}$ we have $q_{j}^{(l)}\left(z_{j}\right)=0$ for $l \in\left\{0, \ldots, \mathfrak{d}_{q_{j}}\left(z_{j}\right)-1\right\}$ and $q_{j}^{\left(\mathfrak{d}_{q_{j}}\left(z_{j}\right)\right)}\left(z_{j}\right) \neq 0$. Thus,

- $q_{j}(\underline{z})=q_{j}\left(z_{j}\right)$ for $\underline{z} \in \sigma(\Theta(\underline{A})) \backslash Z_{\underline{q}}^{\mathbb{R}}$,

- $q_{j \mathcal{M}}(\underline{z})=0 \in \mathbb{C}^{I(\underline{z})}$ for $\underline{z} \in Z_{\underline{q}}^{\mathrm{i}}$ and

- $q_{j \mathcal{M}}(\underline{z})=\left(q_{j \mathcal{M}}(\underline{z})_{\alpha}\right)_{\alpha \in I(\underline{z})}$ with

$$
q_{j \mathcal{M}}(\underline{z})_{\alpha}= \begin{cases}0, & \text { if } \alpha \in I(\underline{z}) \backslash\left\{\mathfrak{d}_{q_{j}}\left(z_{j}\right) e_{j}\right\}, \\ \frac{1}{\mathfrak{d}_{q_{j}}\left(z_{j}\right) !} q_{j}^{\left(\mathfrak{d}_{q_{j}}\left(z_{j}\right)\right)}\left(z_{j}\right), & \text { if } \alpha=\mathfrak{d}_{q_{j}}\left(z_{j}\right) e_{j} .\end{cases}
$$

for $\underline{z} \in Z_{\underline{q}}^{\mathbb{R}}$; see Example 5.1. $\diamond$

Lemma 5.10. For every $\phi \in \mathcal{M}$ there exists an $s \in \mathbb{C}\left[z_{1}, \ldots, z_{n}\right]$ such that $\phi(\underline{w})-s_{\mathcal{M}}(\underline{w})=0$ for all $\underline{w} \in Z_{\underline{q}}$, such that $\phi \mapsto s$ is linear and such that $s=0$ if $\phi(\underline{w})=0$ for all $\underline{w} \in Z_{\underline{q}}$.

Proof. For $\underline{w} \in Z_{\underline{q}}$ the polynomial

$$
p^{\underline{w}}(\underline{z}):=\prod_{v \in Z_{\underline{q}} \backslash\{\underline{w}\}} \prod_{\substack{j=1 \\ v_{j} \neq w_{j}}}^{n}\left(z_{j}-v_{j}\right)^{\mathfrak{d}_{q_{j}}\left(v_{j}\right)+1} \in \mathbb{C}\left[z_{1}, \ldots, z_{n}\right]
$$

satisfies $D^{\alpha} p^{\underline{w}}(\underline{v})=0$ for $\underline{v} \in Z_{\underline{q}} \backslash\{\underline{w}\}$ and $\alpha \in\left[0, \mathfrak{d}_{\underline{q}}(\underline{v})\right]$ as can be checked with the help of the multivariable Leibniz rule. Moreover, $p \underline{w}(\underline{w}) \neq 0$. As noted in Remark 5.3

$$
\left(\frac{1}{\alpha !} D^{\alpha} p^{\underline{w}}(\underline{w})\right)_{\alpha \in\left[0, \mathfrak{d}_{q}(\underline{w})\right]} \in \mathbb{C}^{\left[0, \mathfrak{d}_{\underline{q}}(\underline{w})\right]}
$$

has a multiplicative inverse $b \in \mathbb{C}^{\left[0, \mathfrak{d}_{\underline{q}}(\underline{w})\right]}$. Let $a \in \mathbb{C}^{\left[0, \mathfrak{d}_{\underline{q}}(\underline{w})\right]}$ be given by $a_{\alpha}=\phi(\underline{w})_{\alpha}$ for all $\alpha \in I(\underline{w})$ and $a_{\alpha}=0$ for $\alpha \in\left[0, \mathfrak{d}_{q}(\underline{w})\right] \backslash I(\underline{w})$ and set

$$
r^{\underline{w}}(\underline{z}):=\left(\sum_{\alpha \in\left[0, \mathfrak{d}_{\underline{q}}(\underline{w})\right]}(a \cdot b)_{\alpha}(\underline{z}-\underline{w})^{\alpha}\right) \cdot p^{\underline{w}}(\underline{z}) .
$$

Using again the multivariable Leibniz rule we derive $D^{\alpha} r \underline{w}(\underline{v})=0$ for $\underline{v} \in$ $Z_{\underline{q}} \backslash\{\underline{w}\}, \alpha \in\left[0, \mathfrak{d}_{\underline{q}}(\underline{v})\right]$, and

$$
\left(\frac{1}{\alpha !} D^{\alpha} r^{\underline{w}}(\underline{w})\right)_{\alpha \in\left[0, \mathfrak{d}_{\underline{q}}(\underline{w})\right]}=(a \cdot b) \cdot\left(\frac{1}{\alpha !} D^{\alpha} p^{\underline{w}}(\underline{w})\right)_{\alpha \in\left[0, \mathfrak{d}_{\underline{q}}(\underline{w})\right]}=a .
$$


From this we derive for $s(\underline{z}):=\sum_{\underline{v} \in Z_{\underline{q}}} r \underline{v}(\underline{z})$

$$
\begin{aligned}
s_{\mathcal{M}}(\underline{w}) & =\sum_{\underline{v} \in Z_{\underline{q}}} r \frac{v}{\mathcal{M}}(\underline{w})=r \frac{w}{\mathcal{M}}(\underline{w}) \\
& =\pi_{\left[0, \mathfrak{d}_{\underline{q}}(\underline{w})\right], I(\underline{w})}\left(\frac{1}{\alpha !} D^{\alpha} r^{\underline{w}}(\underline{w})\right)_{\alpha \in\left[0, \mathfrak{d}_{\underline{q}}(\underline{w})\right]}=\phi(\underline{w}) .
\end{aligned}
$$

Finally, by our choice of $a \in \mathbb{C}^{\left[0, \mathfrak{d}_{\underline{q}}(\underline{w})\right]}$ for each $\underline{w} \in Z_{\underline{q}}$ the polynomial $s$ depends linearly on $\phi$ and $\phi(\underline{w})=0$ yields $a=0$ for all $\underline{w} \in Z_{\underline{q}}$ which implies $s=0$.

Remark 5.11. The polynomial $s \in \mathbb{C}\left[z_{1}, \ldots, z_{n}\right]$ constructed in the proof of Lemma 5.10 only depends on $\phi(\underline{w}) \in \mathbb{C}^{I(\underline{w})}, \underline{w} \in Z_{\underline{q}}$. Moreover, by construction the degree of $s$ is at most

$$
d:=\max _{\underline{v} \in Z_{\underline{q}} \backslash\{\underline{w}\}}\left|\mathfrak{d}_{\underline{q}}(\underline{v})\right| \cdot\left(\sum_{\underline{v} \in Z_{\underline{q}} \backslash\{\underline{w}\}}\left(\left|\mathfrak{d}_{\underline{q}}(\underline{v})\right|+n\right)\right) .
$$

It is easy to see from the previous proof that the coefficients $\left(\frac{1}{\alpha !} D^{\alpha} s(0)\right)_{|\alpha| \leq d}$ of $s$ depend linearly and continuously on $\phi$, when $\mathcal{M}$ is provided with the seminorm

$$
\max _{\underline{w} \in Z_{\underline{q}}} \max _{\alpha \in I(\underline{w})}\left|\phi(\underline{w})_{\alpha}\right|,
$$

which implies

$$
\max _{|\alpha| \leq d} \frac{1}{\alpha !}\left|D^{\alpha} s(0)\right| \leq C \cdot \max _{\underline{w} \in Z_{\underline{q}}} \max _{\alpha \in I(\underline{w})}\left|\phi(\underline{w})_{\alpha}\right|
$$

for some $C>0$. $\diamond$

Corollary 5.12. For every $\phi \in \mathcal{M}$ and every $s \in \mathbb{C}\left[z_{1}, \ldots, z_{n}\right]$ such that $\phi(\underline{w})-s_{\mathcal{M}}(\underline{w})=0$ for all $\underline{w} \in Z_{q}$ there exists a function $g: \sigma(\Theta(\underline{A})) \cup Z_{q} \rightarrow \mathbb{C}$ satisfying $\left.g\right|_{Z_{\underline{q}}} \equiv 0$ such that ${ }^{6}$

$$
\phi(\underline{z})=s_{\mathcal{M}}(\underline{z})+g(\underline{z}) \cdot\left(\sum_{j=1}^{n}\left(q_{j}\right)_{\mathcal{M}}(\underline{z})\right)
$$

for all $\underline{z} \in \sigma(\Theta(\underline{A})) \cup Z_{\underline{q}}$.

Proof. For $\underline{z} \in \sigma(\Theta(\underline{A}))$ we know from Proposition 4.2 that $\sum_{j=1}^{n} q_{j}\left(z_{j}\right)=0$ implies $\underline{z} \in Z_{\underline{q}}$. Therefore, if $s \in \mathbb{C}\left[z_{1}, \ldots, z_{n}\right]$ is as in Lemma 5.10, then setting

$$
g(\underline{z}):= \begin{cases}\frac{1}{\sum_{j=1}^{n} q_{j}\left(z_{j}\right)} \cdot(\phi(\underline{z})-s(\underline{z})), & \text { if } \underline{z} \in \sigma(\Theta(\underline{A})) \backslash Z_{\underline{q}}^{\mathbb{R}}, \\ 0, & \text { if } \underline{z} \in Z_{\underline{q}},\end{cases}
$$

we obtain a well defined function $g: \sigma(\Theta(\underline{A})) \cup Z_{\underline{q}} \rightarrow \mathbb{C}$ with the desired properties.

\footnotetext{
${ }^{6}$ Here $g(\underline{z}) \cdot(\ldots)$ denotes the scalar multiplication of $g(\underline{z}) \in \mathbb{C}$ with a vector from $\mathbb{C}^{I(\underline{z})}$.
} 
Definition 5.13. With the notation from Definition 5.5 we denote by $\mathcal{F}$ the set of all $\phi \in \mathcal{M}$ such that $\left.\phi\right|_{\sigma(\Theta(\underline{A})) \backslash Z_{\underline{q}}^{\mathbb{R}}}$ as a mapping from $\sigma(\Theta(\underline{A})) \backslash Z_{\underline{q}}^{\mathbb{R}}$ to $\mathbb{C}$ is Borel measurable and bounded, and such that for each $\underline{w} \in \sigma(\Theta(\underline{A})) \cap Z_{\underline{q}}^{\mathbb{R}}$, which is not isolated in $\sigma(\Theta(\underline{A}))$,

$$
\frac{\phi(\underline{z})-\sum_{\alpha \in\left[0, \mathfrak{d}_{q}(\underline{w})\right)}(\phi(\underline{w}))_{\alpha}(\underline{z}-\underline{w})^{\alpha}}{\max _{j=1, \ldots, n}\left|z_{j}-w_{j}\right|^{\mathfrak{d}_{q_{j}}\left(w_{j}\right)}}
$$

is bounded for $\underline{z} \in \sigma(\Theta(\underline{A})) \cap U(\underline{w}) \backslash\{\underline{w}\}$, where $U(\underline{w})$ is a sufficiently small neighborhood of $\underline{w} . \diamond$

Using Big $O$ notation, the fact that (5.3) is bounded on a sufficiently small neighborhood of $w$ can equivalently be expressed as

$$
\phi(\underline{z})=\sum_{\alpha \in\left[0, \mathfrak{d}_{\underline{q}}(\underline{w})\right)}(\phi(\underline{w}))_{\alpha}(\underline{z}-\underline{w})^{\alpha}+O\left(\max _{j=1, \ldots, n}\left|z_{j}-w_{j}\right|^{\mathfrak{d}_{q_{j}}\left(w_{j}\right)}\right)
$$

as $\sigma(\Theta(\underline{A})) \backslash Z_{\underline{q}}^{\mathbb{R}} \ni \underline{z} \rightarrow \underline{w}$.

Remark 5.14. Since (5.3) is bounded for $\underline{z} \in\left(\sigma(\Theta(\underline{A})) \backslash Z_{\underline{q}}^{\mathbb{R}}\right) \backslash U(\underline{w})$ if $\left.\phi\right|_{\sigma(\Theta(\underline{A})) \backslash Z_{\underline{q}}^{\mathbb{R}}}$ is bounded, a function $\phi \in \mathcal{M}$ belongs to $\mathcal{F}$ if and only if $\phi(\underline{z})$ and (5.3) for all non isolated $\underline{w} \in \sigma(\Theta(\underline{A})) \cap Z_{\underline{q}}^{\mathbb{R}}$ are bounded as $\underline{z}$ runs in

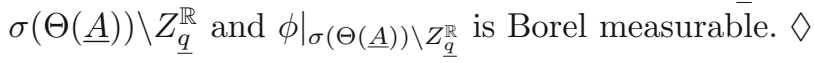

Remark 5.15. It is straight forward to check that $\mathcal{F}+\mathcal{F} \subseteq \mathcal{F}, \mathbb{C} \cdot \mathcal{F} \subseteq \mathcal{F}$, and $\mathcal{F}^{\#} \subseteq \mathcal{F}$. In fact, equalities prevail. We also have $\mathcal{F} \cdot \mathcal{F} \subseteq \mathcal{F}$.

Indeed, if $\phi, \psi \in \mathcal{F}$, then $\left.(\phi \cdot \psi)\right|_{\sigma(\Theta(\underline{A})) \backslash Z_{q}^{\mathbb{R}}}$ is clearly measurable and bounded. Moreover, for any $\alpha \in\left(\mathbb{N}_{0}\right)^{n}$ and $\beta \in\left(\mathbb{N}_{0}\right)^{n} \backslash\left[0, \mathfrak{d}_{\underline{q}}(\underline{w})\right)$ we have

$$
\begin{aligned}
\left(O\left(\max _{j=1, \ldots, n}\left|z_{j}-w_{j}\right|^{\mathfrak{d}_{q_{j}}\left(w_{j}\right)}\right)\right)^{2} & =O\left(\max _{j=1, \ldots, n}\left|z_{j}-w_{j}\right|^{\mathfrak{d}_{q_{j}}\left(w_{j}\right)}\right), \\
(\underline{z}-\underline{w})^{\alpha} \cdot O\left(\max _{j=1, \ldots, n}\left|z_{j}-w_{j}\right|^{\mathfrak{d}_{q_{j}}\left(w_{j}\right)}\right) & =O\left(\max _{j=1, \ldots, n}\left|z_{j}-w_{j}\right|^{\mathfrak{d}_{q_{j}}\left(w_{j}\right)}\right), \\
(\underline{z}-\underline{w})^{\beta} & =O\left(\max _{j=1, \ldots, n}\left|z_{j}-w_{j}\right|^{\mathfrak{d}_{q_{j}}\left(w_{j}\right)}\right)
\end{aligned}
$$

as $\underline{z} \rightarrow \underline{w}$. For a not isolated $\underline{w} \in \sigma(\Theta(\underline{A})) \cap Z_{\underline{q}}^{\mathbb{R}}(5.4)$ therefore yields

$$
\begin{aligned}
\phi(\underline{z}) \cdot \psi(\underline{z})= & \left(\sum_{\alpha \in\left[0, \mathfrak{d}_{\underline{q}}(\underline{w})\right)}(\phi(\underline{w}))_{\alpha}(\underline{z}-\underline{w})^{\alpha}+O\left(\max _{j=1, \ldots, n}\left|z_{j}-w_{j}\right|^{\mathfrak{d}_{q_{j}}\left(w_{j}\right)}\right)\right) \\
& \cdot\left(\sum_{\alpha \in\left[0, \mathfrak{d}_{\underline{q}}(\underline{w})\right)}(\psi(\underline{w}))_{\alpha}(\underline{z}-\underline{w})^{\alpha}+O\left(\max _{j=1, \ldots, n}\left|z_{j}-w_{j}\right|^{\left.\mathfrak{d}_{q_{j}}\left(w_{j}\right)\right)}\right)\right) \\
= & \left.\sum_{\alpha \in\left(\mathbb{N}_{0}\right)^{n}}\left(\sum_{\beta, \gamma \in\left[0, \mathfrak{o}_{\underline{q}}(\underline{w})\right)}(\phi(\underline{w}))_{\beta} \cdot(\psi(\underline{w}))_{\gamma}\right) \cdot(\underline{z}-\underline{w})^{\alpha}\right)
\end{aligned}
$$




$$
\begin{aligned}
& +O\left(\max _{j=1, \ldots, n}\left|z_{j}-w_{j}\right|^{\mathfrak{d}_{q_{j}}\left(w_{j}\right)}\right) \\
= & \sum_{\alpha \in\left[0, \mathfrak{d}_{\underline{q}}(\underline{w})\right)}(\phi(\underline{w}) \cdot \psi(\underline{w}))_{\alpha}(\underline{z}-\underline{w})^{\alpha}+O\left(\max _{j=1, \ldots, n}\left|z_{j}-w_{j}\right|^{\mathfrak{d}_{q_{j}}\left(w_{j}\right)}\right) .
\end{aligned}
$$

Thus, $\mathcal{F}$ is a $*$-subalgebra of $\mathcal{M}$. $\diamond$

Example 5.16. Let $\underline{w} \in Z_{\underline{q}}$ be an isolated point of $\sigma(\Theta(\underline{A})) \cup Z_{\underline{q}}\left(\subseteq \mathbb{C}^{n}\right)$, let $a \in \mathbb{C}^{I(\underline{w})}$ and let $\delta_{\underline{w}}: \sigma(\Theta(\underline{A})) \cup Z_{q} \rightarrow \mathbb{C}$ defined by $\delta_{\underline{w}}(\underline{w}):=1$ and $\delta_{w}(\underline{z}):=0$ if $\underline{z} \neq \underline{w}$. Then $\delta_{w} a \in \mathcal{M}$ defined by $\left(\delta_{w} a\right)(\underline{z}):=0$ for $\underline{z} \neq \underline{w}$ and by $\left(\delta_{\underline{w}} a\right)(\underline{w})=a$ is an element of $\mathcal{F}$. Clearly, every element of $Z_{q}^{\mathrm{i}}$ is isolated in $\sigma(\Theta(\underline{A})) \cup Z_{\underline{q}} . \diamond$

Lemma 5.17. Let $f: \operatorname{dom} f \rightarrow \mathbb{C}$ be a function with the properties mentioned in Definition 5.7. If $\left.f\right|_{\sigma(\Theta(\underline{A}))}$ is bounded and measurable, then $f_{\mathcal{M}} \in \mathcal{F}$.

Proof. Under the present assumption $\left.f_{\mathcal{M}}\right|_{\sigma(\Theta(\underline{A})) \backslash Z_{\underline{q}}^{\mathbb{R}}}$ as a mapping from $\sigma(\Theta(\underline{A})) \backslash Z_{\underline{q}}^{\mathbb{R}}$ to $\mathbb{C}$ coincides with $\left.f\right|_{\sigma(\Theta(\underline{A})) \backslash Z_{\underline{q}}^{\mathbb{R}}}$ and is therefore bounded and measurable.

Since for a fixed $w \in \sigma(\Theta(\underline{A})) \cap Z_{q}^{\mathbb{R}}$ which is non-isolated in $\sigma(\Theta(\underline{A}))$ the function $f$ is $m:=\max _{\zeta \in Z_{q}^{\mathbb{R}}}\left|\mathfrak{d}_{q}(\zeta)\right|-n+1$ times continuous differentiable on an open subset of $\mathbb{R}^{n}$ containing $\underline{w}$, by the Taylor Approximation Theorem from multidimensional calculus the expression

$$
f(\underline{z})-\sum_{\substack{\alpha \in\left(\mathbb{N}_{0}\right)^{n} \\|\alpha| \leq m-1}} \frac{1}{\alpha !} D^{\alpha} f(\underline{w})(\underline{z}-\underline{w})^{\alpha}
$$

is a $O\left(\|\underline{z}-\underline{w}\|_{\infty}^{m}\right)$ as $\underline{z} \rightarrow \underline{w}$. Because of $\mathfrak{d}_{q_{j}}\left(w_{j}\right) \geq 1$ we have $\left|\mathfrak{d}_{q}(\underline{w})\right|-n+1 \geq$ $\mathfrak{d}_{q_{j}}(w)$ for all $j=1, \ldots, n$, and hence

$$
\|\underline{z}-\underline{w}\|_{\infty}^{m} \leq \max _{j=1, \ldots, n}\left|z_{j}-w_{j}\right|^{\left|\mathfrak{d}_{\underline{q}}(\underline{w})\right|-n+1} \leq \max _{j=1, \ldots, n}\left|z_{j}-w_{j}\right|^{\mathfrak{d}_{q_{j}}\left(w_{j}\right)}
$$

for $\|z-w\|_{\infty} \leq 1$. Moreover, for $\alpha \in\left(\mathbb{N}_{0}\right)^{n} \backslash\left[0, \mathfrak{d}_{\underline{q}}(\underline{w})\right)$ we infer $\alpha_{k} \geq \mathfrak{d}_{q_{k}}(w)$ for some $k \in\{1, \ldots, n\}$, and in turn

$$
\left|(\underline{z}-\underline{w})^{\alpha}\right| \leq\left|z_{k}-w_{k}\right|_{k}^{\alpha} \leq\left|z_{k}-w_{k}\right|^{\mathfrak{d}_{q_{k}}(w)} \leq \max _{j=1, \ldots, n}\left|z_{j}-w_{j}\right|^{\mathfrak{d}_{q_{j}}(w)}
$$

for $\|\underline{z}-\underline{w}\|_{\infty} \leq 1$. Therefore,

$$
\begin{aligned}
& \mid f_{\mathcal{M}}(\underline{z})- \sum_{\substack{\alpha \in\left[0, \mathfrak{d}_{\underline{q}}(\underline{w})\right) \\
\leq}}\left(f_{\mathcal{M}}(\underline{w})\right)_{\alpha}(\underline{z}-\underline{w})^{\alpha} \mid \\
& \leq\left|f(\underline{z})-\sum_{\substack{\alpha \in\left(\mathbb{N}_{0}\right)^{n} \\
|\alpha| \leq m-1}} \frac{1}{\alpha !} D^{\alpha} f(\underline{w})(\underline{z}-\underline{w})^{\alpha}\right| \\
&+\sum_{\substack{\alpha \in\left(\mathbb{N}_{0}\right)^{n} \backslash\left[0, \mathfrak{d}_{\underline{q}}(\underline{w})\right) \\
|\alpha| \leq m-1}} \frac{1}{\alpha !}\left|D^{\alpha} f(\underline{w})\right| \cdot\left|(\underline{z}-\underline{w})^{\alpha}\right|
\end{aligned}
$$


is a $O\left(\max _{j=1, \ldots, n}\left|z_{j}-w_{j}\right|^{\mathfrak{d}_{q_{j}}\left(w_{j}\right)}\right)$ as $\underline{z} \rightarrow \underline{w}$. According to Definition 5.13 the function $f_{\mathcal{M}}$ then belongs to $\mathcal{F}$.

Lemma 5.18. If $\phi \in \mathcal{F}$ is such that $\phi(\underline{z})$ is invertible in $\mathbb{C}^{I(\underline{z})}$ for all $\underline{z} \in$ $\sigma(\Theta(\underline{A})) \cup Z_{\underline{q}}$ and such that 0 does not belong to the closure of $\phi\left(\sigma(\Theta(\underline{A})) \backslash Z_{\underline{q}}^{\mathbb{R}}\right)$ as a subset of $\mathbb{C}$, then $\phi^{-1}$ defined by $\phi^{-1}(\underline{z}):=\phi(\underline{z})^{-1}$ also belongs to $\mathcal{F}$.

Proof. By the first assumption $\phi^{-1}$ is a well-defined function belonging to $\mathcal{M}$. Since 0 does not belong to the closure of $\phi\left(\sigma(\Theta(\underline{A})) \backslash Z_{\underline{q}}^{\mathbb{R}}\right)$ the mapping $\underline{z} \mapsto \frac{1}{\phi(\underline{z})}$ is bounded on $\sigma(\Theta(\underline{A})) \backslash Z_{\underline{q}}^{\mathbb{R}}$. The measurability of $\left.\phi\right|_{\sigma(\Theta(\underline{A})) \backslash Z_{\underline{q}}^{\mathbb{R}}}$ clearly implies the measurability of $\underline{z} \mapsto \frac{1}{\phi(\underline{z})}$ on $\sigma(\Theta(\underline{A})) \backslash Z_{\underline{q}}^{\mathbb{R}}$.

Let $\underline{w} \in \sigma(\Theta(\underline{A})) \cap Z_{\underline{q}}^{\mathbb{R}}$ be non-isolated in $\sigma(\Theta(\underline{A}))$. For $\underline{z} \in \sigma(\Theta(\underline{A})) \backslash Z_{\underline{q}}^{\mathbb{R}}$ we calculate

$$
\begin{aligned}
\phi^{-1}(\underline{z})-\sum_{\alpha \in\left[0, \mathfrak{d}_{\underline{q}}(\underline{w})\right)}\left(\phi^{-1}(\underline{w})\right)_{\alpha}(\underline{z}-\underline{w})^{\alpha} \\
=\frac{1}{\phi(\underline{z})}-\frac{1}{\sum_{\alpha \in\left[0, \mathfrak{d}_{\underline{q}}(\underline{w})\right)}(\phi(\underline{w}))_{\alpha}(\underline{z}-\underline{w})^{\alpha}} \\
\quad+\frac{1}{\sum_{\alpha \in\left[0, \mathfrak{d}_{\underline{q}}(\underline{w})\right)}(\phi(\underline{w}))_{\alpha}(\underline{z}-\underline{w})^{\alpha}} \\
\quad-\sum_{\alpha \in\left[0, \mathfrak{d}_{\underline{q}}(\underline{w})\right)}\left(\phi^{-1}(\underline{w})\right)_{\alpha}(\underline{z}-\underline{w})^{\alpha} .
\end{aligned}
$$

The term (5.6) can be rewritten as

$$
-\frac{1}{\phi(\underline{z})} \cdot \frac{1}{\sum_{\alpha \in\left[0, \mathfrak{d}_{\underline{q}}(\underline{w})\right)}(\phi(\underline{w}))_{\alpha}(\underline{z}-\underline{w})^{\alpha}} \cdot\left(\phi(\underline{z})-\sum_{\alpha \in\left[0, \mathfrak{d}_{\underline{q}}(\underline{w})\right)}(\phi(\underline{w}))_{\alpha}(\underline{z}-\underline{w})^{\alpha}\right) .
$$

By assumption $\frac{1}{\phi(\underline{z})}$ is bounded. The invertibility of $\phi(\underline{w})$ guarantees $\left(\phi(\underline{w})^{-1}\right)_{0} \neq 0$, which yields the boundedness of

$$
\frac{1}{\sum_{\alpha \in\left[0, \mathfrak{d}_{\underline{q}}(\underline{w})\right)}(\phi(\underline{w}))_{\alpha}(\underline{z}-\underline{w})^{\alpha}}
$$

on a certain neighborhood of $\underline{w}$. From $\phi \in \mathcal{F}$ we infer $\phi(\underline{z})-\sum_{\alpha \in\left[0, \mathfrak{d}_{q}(\underline{w})\right)}$ $(\phi(\underline{w}))_{\alpha}(\underline{z}-\underline{w})^{\alpha}=O\left(\max _{j=1, \ldots, n}\left|z_{j}-w_{j}\right|^{\mathfrak{d}_{q_{j}}\left(w_{j}\right)}\right)$ as $\underline{z} \rightarrow \underline{w}$. Thus, (5.6) is also an $O\left(\max _{j=1, \ldots, n}\left|z_{j}-w_{j}\right|^{\mathfrak{d}_{q_{j}}\left(w_{j}\right)}\right)$.

We can write (5.7) as (5.8) times

$$
\begin{aligned}
1-\sum_{\alpha \in\left(\mathbb{N}_{0}\right)^{n}} \sum_{\substack{\beta, \gamma \in\left[0, \mathfrak{d}_{\underline{q}}(\underline{w})\right) \\
\beta+\gamma=\alpha}}(\phi(\underline{w}))_{\beta}\left(\phi(\underline{w})^{-1}\right)_{\gamma}(\underline{z}-\underline{w})^{\alpha} \\
=1-\sum_{\alpha \in\left[0, \mathfrak{d}_{\underline{q}}(\underline{w})\right)} \underbrace{\sum_{\substack{\beta, \gamma \in\left[0, \mathfrak{d}_{q}(\underline{w})\right) \\
\beta+\gamma=\alpha}}(\phi(\underline{w}))_{\beta}\left(\phi(\underline{w})^{-1}\right)_{\gamma}(\underline{z}-\underline{w})^{\alpha}}_{=e_{\alpha}}
\end{aligned}
$$




$$
-\sum_{\substack{\alpha \in\left(\mathbb{N}_{0}\right)^{n} \\ \alpha \notin\left[0, \mathfrak{d}_{\underline{q}}(\underline{w})\right)}} \sum_{\substack{\beta, \gamma \in\left[0, \mathfrak{d}_{q}(\underline{w})\right) \\ \beta+\gamma=\alpha}}(\phi(\underline{w}))_{\beta}\left(\phi(\underline{w})^{-1}\right)_{\gamma}(\underline{z}-\underline{w})^{\alpha} .
$$

For $\alpha \in\left(\mathbb{N}_{0}\right)^{n} \backslash\left[0, \mathfrak{d}_{q}(\underline{w})\right)$ we have $\left|(\underline{z}-\underline{w})^{\alpha}\right|=O\left(\max _{j=1, \ldots, n}\left|z_{j}-w_{j}\right|^{\mathfrak{d}_{q_{j}}\left(w_{j}\right)}\right)$; see (5.5). Since $\sum_{\alpha \in\left[0, \mathfrak{o}_{q}(\underline{w})\right)} e_{\alpha}(\underline{z}-\underline{w})^{\alpha}=1$, we see that (5.7) is an $O\left(\max _{j=1, \ldots, n}\left|z_{j}-w_{j}\right|^{\mathfrak{d}_{q_{j}}\left(w_{j}\right)}\right)$. Consequently, $\phi^{-1} \in \mathcal{F}$.

Finally, we can bring a refinement of Corollary 5.12 for functions in $\mathcal{F}$.

Lemma 5.19. Let $\phi \in \mathcal{F}$ be decomposed as

$$
\phi=s_{\mathcal{M}}+g \cdot\left(\sum_{j=1}^{n}\left(q_{j}\right)_{\mathcal{M}}\right)
$$

with $s \in \mathbb{C}\left[z_{1}, \ldots, z_{n}\right]$ and a function $g: \sigma(\Theta(\underline{A})) \cup Z_{\underline{q}} \rightarrow \mathbb{C}$ satisfying $\left.g\right|_{Z_{\underline{q}}} \equiv 0$ as in Corollary 5.12. We will call such a pair $s, g$ an admissible decomposition of $\phi$.

Then $\left.g\right|_{\sigma(\Theta(\underline{A})) \backslash Z_{\underline{q}}^{\mathbb{R}}}$ is bounded and measurable.

Proof. According to Corollary 5.12 there exist decompositions as in (5.9).

By (5.9) and the fact that $\sum_{j=1}^{n} q_{j \mathcal{M}}(\underline{z})$ does not vanish on $\sigma(\Theta(\underline{A})) \backslash Z_{\underline{q}}^{\mathbb{R}}$ (see Proposition 4.2) the measurability of $\left.g\right|_{\sigma(\Theta(\underline{A})) \backslash Z_{\underline{q}}^{\mathbb{R}}}$ follows from the assumed measurability of $\left.\phi\right|_{\sigma(\Theta(\underline{A})) \backslash Z_{\underline{q}}^{\mathbb{R}}}$ for functions $\phi \in \mathcal{F}$; see Definition 5.13.

In order to show the boundedness of $g$, first recall from Proposition 4.2 that

$$
\frac{\max _{j=1, \ldots, n}\left|q_{j}(\underline{z})\right|}{\left|\sum_{j=1}^{n} q_{j}(\underline{z})\right|} \leq \max _{j=1, \ldots, n}\left\|R_{j} R_{j}^{*}\right\|
$$

for $\underline{z} \in \sigma(\Theta(\underline{A})) \backslash Z_{\underline{q}}^{\mathbb{R}}$. Hence,

$$
|g(\underline{z})|=\frac{|\phi(\underline{z})-s(\underline{z})|}{\left|\sum_{j=1}^{n} q_{j}(\underline{z})\right|}=\frac{|\phi(\underline{z})-s(\underline{z})|}{\max _{j=1, \ldots, n}\left|q_{j}\left(z_{j}\right)\right|} \cdot \frac{\max _{j=1, \ldots, n}\left|q_{j}(\underline{z})\right|}{\left|\sum_{j=1}^{n} q_{j}(\underline{z})\right|}
$$

is bounded, if we can prove that the first factor on the right hand side is bounded.

For a fixed non-isolated $\underline{w} \in \sigma(\Theta(\underline{A})) \cap Z_{\underline{q}}^{\mathbb{R}}$ we have

$$
\begin{aligned}
& \frac{|\phi(\underline{z})-s(\underline{z})|}{\max _{j=1, \ldots, n}\left|q_{j}\left(z_{j}\right)\right|} \\
& =\frac{|\phi(\underline{z})-s(\underline{z})|}{\max _{j=1, \ldots, n}\left|z_{j}-w_{j}\right|^{\mathfrak{d}_{q_{j}}\left(w_{j}\right)}} \frac{\max _{j=1, \ldots, n}\left|z_{j}-w_{j}\right|^{\mathfrak{d}_{q_{j}}\left(w_{j}\right)}}{\max _{j=1, \ldots, n}\left|q_{j}\left(z_{j}\right)\right|}
\end{aligned}
$$

Since $w_{j}$ is a zero of $q_{j}$ with multiplicity exactly $\mathfrak{d}_{q_{j}}\left(w_{j}\right)$ for $j=1, \ldots, n$, $\left|z_{j}-w_{j}\right|^{\mathfrak{d}_{q_{j}}\left(w_{j}\right)}=O\left(q_{j}\left(z_{j}\right)\right)$ as $z_{j} \rightarrow w_{j}$, and in turn

$$
\max _{j=1, \ldots, n}\left|z_{j}-w_{j}\right|^{\mathfrak{d}_{q_{j}}\left(w_{j}\right)}=O\left(\max _{j=1, \ldots, n}\left|q_{j}\left(z_{j}\right)\right|\right)
$$


as $\underline{z} \rightarrow \underline{w}$. Hence, the second factor on the right hand side in (5.12) is bounded on a neighborhood of $\underline{w}$. $\phi(\underline{z})-s(\underline{z})$ for $\sigma(\Theta(\underline{A})) \backslash Z_{\underline{q}}^{\mathbb{R}}$ is the difference of

$$
\phi(\underline{z})-\sum_{\alpha \in\left[0, \mathfrak{d}_{\underline{q}}(\underline{w})\right)}(\phi(\underline{w}))_{\alpha}(\underline{z}-\underline{w})^{\alpha}
$$

and

$$
s_{\mathcal{M}}(\underline{z})-\sum_{\alpha \in\left[0, \mathfrak{d}_{\underline{q}}(\underline{w})\right)}(\phi(\underline{w}))_{\alpha}(\underline{z}-\underline{w})^{\alpha} .
$$

Since according to (5.9) we have $s_{\mathcal{M}}(\underline{w})=\phi(\underline{w})$, we conclude from Lemmas 5.17 and $(5.4)$ that $\phi(\underline{z})-s(\underline{z})=O\left(\max _{j=1, \ldots, n}\left|z_{j}-w_{j}\right|^{\mathfrak{d}_{q_{j}}\left(w_{j}\right)}\right)$ as $\sigma(\Theta(\underline{A})) \backslash Z_{q}^{\mathbb{R}} \ni \underline{z} \rightarrow \underline{w}$. Thus, also the first factor on the right hand side in (5.12) is bounded on a neighborhood of $\underline{w}$.

Employing this for any non-isolated $\underline{w} \in \sigma(\Theta(\underline{A})) \cap Z_{\underline{q}}^{\mathbb{R}}$, for each $\underline{w} \in Z_{\underline{q}}^{\mathbb{R}}$ we obtain a neighborhood $U_{\underline{w}}$ of $\underline{w}$ such that (5.12) is bounded on $\sigma(\Theta(\underline{A})) \hat{\cap}$ $\bigcup_{\underline{w} \in Z_{\underline{q}}^{\mathbb{R}}}\left(U_{\underline{w}} \backslash\{\underline{w}\}\right)$. The boundedness on $\sigma(\Theta(\underline{A})) \backslash \bigcup_{\underline{w} \in Z_{\underline{q}}^{\mathbb{R}}} U_{\underline{w}}$ follows from the assumed boundedness of $\left.\phi\right|_{\sigma(\Theta(\underline{A})) \backslash Z_{\underline{q}}^{\mathbb{R}}}$ for functions $\phi \in \mathcal{F}$.

Finally, we want to provide $\mathcal{F}$ with the norm

$$
\begin{aligned}
& \|\phi\|_{\mathcal{F}}:=\max _{\underline{z} \in \sigma(\Theta(\underline{A})) \cup Z_{\underline{q}}}|\phi(\underline{z})| \\
& \quad+\max _{\underline{w} \in Z_{\underline{q}}^{\mathbb{R}} \underline{w} \text { not isolated } \sup _{\underline{z} \in \sigma(\Theta(\underline{A})) \backslash Z_{\underline{q}}^{\mathbb{R}}}} \frac{\phi(\underline{z})-\sum_{\alpha \in\left[0, \mathfrak{d}_{\underline{q}}(\underline{w})\right)}(\phi(\underline{w}))_{\alpha}(\underline{z}-\underline{w})^{\alpha}}{\max _{j=1, \ldots, n}\left|z_{j}-w_{j}\right|^{\mathfrak{d}_{q_{j}}\left(w_{j}\right)}} \mid,
\end{aligned}
$$

where $|\phi(\underline{z})|=\max _{\alpha \in I(\underline{z})}\left|\phi(\underline{z})_{\alpha}\right|$ for $\underline{z} \in Z_{\underline{q}}$. Using Remark 5.14 it is straight forward to check that $\|\cdot\|_{\mathcal{F}}$ is finitely valued and is indeed a norm.

Lemma 5.20. The mapping $\mathcal{F} \ni \phi \mapsto s_{\mathcal{M}} \in \mathcal{F}$, which assigns to $\phi$ the polynomial $s \in \mathbb{C}\left[z_{1}, \ldots, z_{n}\right]$ from Lemma 5.10 , is linear and bounded when $\mathcal{F}$ is provided with $\|\cdot\|_{\mathcal{F}}$. Moreover, the mapping ${ }^{7}$

$$
\mathcal{F} \ni \phi \mapsto g \in \mathcal{B}\left(\sigma(\Theta(\underline{A})) \cup Z_{\underline{q}}^{\mathbb{R}}, \mathbb{C}\right),
$$

which assigns to $\phi$ the function $g$ from (5.9) where $s \in \mathbb{C}\left[z_{1}, \ldots, z_{n}\right]$ is as in Lemma 5.10, is also linear and bounded.

Proof. Since all norms on a finite dimensional vector spaces are equivalent, it follows from Remark 5.11 the mapping $\mathcal{F} \ni \phi \mapsto s \in \mathbb{C}\left[z_{1}, \ldots, z_{n}\right]_{\leq d}$ is bounded, where $d \in \mathbb{N}$ is as in Remark 5.11 and where $\mathbb{C}\left[z_{1}, \ldots, z_{n}\right]_{\leq d}$ denotes the space of all polynomials in $\mathbb{C}\left[z_{1}, \ldots, z_{n}\right]$ with degree less or equal to $d$. Since linear mappings defined on finite dimensional normed spaces are always bounded, also $\mathbb{C}\left[z_{1}, \ldots, z_{n}\right]_{\leq d} \ni s \mapsto s_{\mathcal{M}} \in \mathcal{F}$ is bounded. Thus, we verified the first part of the present assertion.

For given $\phi \in \mathcal{F}$ and $s \in \mathbb{C}\left[z_{1}, \ldots, z_{n}\right]$ as in Lemma 5.10 the corresponding function $g(\phi, s)$ in $(5.9)$ coincides with $g\left(\phi-s_{\mathcal{M}}, 0\right)$, i.e. the function $g$

\footnotetext{
${ }^{7}$ Here $\mathcal{B}\left(\sigma(\Theta(\underline{A})) \cup Z_{\underline{q}}^{\mathbb{R}}, \mathbb{C}\right)$ denotes the Banach space of all complex valued and bounded functions on $\sigma(\Theta(\underline{A})) \underline{\cup} Z_{\underline{q}}^{\mathbb{R}}$ provided with $\|\cdot\|_{\infty}$.
} 
in (5.9) applied to $\phi-s_{\mathcal{M}} \in \mathcal{F}$ and $0 \in \mathbb{C}\left[z_{1}, \ldots, z_{n}\right]$. Since $\phi \mapsto \phi-s_{\mathcal{M}}$ is linear and bounded by the first part of the proof, it remains to check that $\phi \mapsto g(\phi, 0)$ is linear and bounded on the subspace $\left\{\phi \in \mathcal{F}:\left.\phi\right|_{Z_{\underline{q}}} \equiv 0\right\}$.

Let $\phi \in \mathcal{F}$ with $\left.\phi\right|_{Z_{\underline{q}}} \equiv 0$. By (5.11) and (5.10) we have

$$
|g(\phi, 0)(\underline{z})| \leq \max _{j=1, \ldots, n}\left\|R_{j} R_{j}^{*}\right\| \cdot \frac{|\phi(\underline{z})|}{\max _{j=1, \ldots, n}\left|q_{j}\left(z_{j}\right)\right|} .
$$

Chose $\epsilon>0$ so small that $\|\underline{w}-\underline{v}\|>2 \epsilon$ for two different $\underline{v}, \underline{w} \in \sigma(\Theta(\underline{A})) \cap Z_{\underline{q}}^{\mathbb{R}}$ which are not isolated in $\sigma(\Theta(\underline{A})) \cup Z_{\underline{q}}^{\mathbb{R}}$.

If for $\underline{z} \in \sigma(\Theta(\underline{A})) \backslash Z_{\underline{q}}^{\mathbb{R}}$ we have $\|\underline{z}-\underline{w}\| \geq \epsilon$ for all not isolated $\underline{w} \in \sigma(\Theta(\underline{A})) \cap Z_{q}^{\mathbb{R}}$, then $\max _{j=1, \ldots, n}\left|q_{j}\left(z_{j}\right)\right| \geq \rho$ for some $\rho>0$ which is independent from $\underline{z}$. Hence,

$$
|g(\phi, 0)(\underline{z})| \leq D \cdot|\phi(\underline{z})| \leq D \cdot\|\phi\|_{\mathcal{F}}
$$

for some constant $D>0$ which is also independent from $\underline{z}$. If $\|\underline{z}-\underline{w}\|<\epsilon$ for some not isolated $\underline{w} \in \sigma(\Theta(\underline{A})) \cap Z_{q}^{\mathbb{R}}$, then $\max _{j=1, \ldots, n}\left|z_{j}-w_{j}\right|^{\mathfrak{d}_{j}\left(w_{j}\right)} \leq$ $\eta_{\underline{w}} \max _{j=1, \ldots, n}\left|q_{j}\left(z_{j}\right)\right|$ for some constant $\eta_{\underline{w}}>0$. Hence,

$$
|g(\phi, 0)(\underline{z})| \leq D_{\underline{w}} \cdot \frac{|\phi(\underline{z})|}{\max _{j=1, \ldots, n}\left|z_{j}-w_{j}\right|^{\mathfrak{d}_{q_{j}}\left(w_{j}\right)}} \leq D_{\underline{w}} \cdot\|\phi\|_{\mathcal{F}}
$$

for some constant $D_{\underline{w}}>0$.

\section{The Spectral Theorem}

In the present section we again have a tuple $\underline{A}=\left(A_{j}\right)_{j=1}^{n}$ whose entries are pairwise commuting, bounded, self-adjoint and definitizable operators $A_{1}, \ldots, A_{n} \in L_{b}(\mathcal{K})$ on a Krein space $\mathcal{K}$ where for $j=1, \ldots, n$ we denote by $q_{j} \in \mathbb{R}[\zeta] \backslash\{0\}$ a definitizing polynomial for $A_{j}$. We shall employ the same notation as the previous two sections. In particular, we will again write $\mathcal{H}$ for $\mathcal{H}_{\{1, \ldots, n\}}, T$ for $T_{\{1, \ldots, n\}}, \Theta$ for $\Theta_{\{1, \ldots, n\}}$ and $R_{j}$ for $R_{\{j\} /\{1, \ldots, n\}}$. In addition, we shall write $\Xi$ for $\Xi_{\{1, \ldots n\}}, \Xi_{j}$ for $\Xi_{\{j\}}, E$ for $E_{\{1, \ldots, n\}}$ and $E_{j}$ for $E_{\{j\}}$. We start with an elementary algebraic lemma.

Lemma 6.1. Let $v\left(z_{1}, \ldots, z_{n}\right) \in \mathbb{C}\left[z_{1}, \ldots, z_{n}\right]$ be such that the $z_{j}$-degree of $v$ is less than $\operatorname{deg} q_{j}$ for $j=1, \ldots, n$. If $D^{\alpha} v(\underline{w})=0$ for all $\alpha \in\left[0, \mathfrak{d}_{q}(\underline{w})\right)$ and all $\underline{w} \in Z_{\underline{q}}$, then $v=0$.

Proof. We proof this assertion by induction on $n$. For $n=1$ this is clear. Assume the statement is true for $n-1 \in \mathbb{N}$. If $v\left(z_{1}, \ldots, z_{n}\right) \in \mathbb{C}\left[z_{1}, \ldots, z_{n}\right]$ has the asserted properties, then the polynomial $\frac{\partial^{m}}{\partial z_{n}^{m}} v\left(z_{1}, \ldots, z_{n-1}, w_{n}\right) \in$ $\mathbb{C}\left[z_{1}, \ldots, z_{n-1}\right]$ satisfies the assumption of the present lemma for any $m \in$ $\left\{0, \ldots, \mathfrak{d}_{q_{n}}(\underline{w})-1\right\}$ and any $w_{n} \in Z_{q_{n}}$. By induction hypothesis all these polynomials vanish. Keeping $z_{1}, \ldots, z_{n-1} \in \mathbb{C}$ fixed this implies that $v\left(z_{1}, \ldots\right.$, $\left.z_{n-1}, z_{n}\right)$ as a polynomial in the variable $z_{n}$ vanishes. 
Lemma 6.2. For a given $\phi \in \mathcal{F}$ and two admissible decompositions $s, g$ and $r, h$ of $\phi$ in the sense of Lemma 5.19 we have

$$
s(\underline{A})+\Xi\left(\int_{\sigma(\Theta(\underline{A}))} g d E\right)=r(\underline{A})+\Xi\left(\int_{\sigma(\Theta(\underline{A}))} h d E\right),
$$

where $E$ denotes the common spectral measure of $\Theta(\underline{A}) \in L_{b}(\mathcal{H})^{n}$ on the Borel-subsets of $\mathbb{R}^{n}$; see Theorem 2.2 and Remark 2.3 .

Proof. By assumption $\phi-s_{\mathcal{M}}, \phi-r_{\mathcal{M}}$ and in consequence also their difference $s_{\mathcal{M}}-r_{\mathcal{M}}$ vanish at all points of $Z_{\underline{q}}$. Considering $p(\underline{z}):=s(\underline{z})-$ $r(\underline{z}) \in \mathbb{C}\left[z_{1}, \ldots, z_{n}\right]$ for fixed $z_{2}, \ldots, z_{n}$ as a polynomial in $\mathbb{C}\left[z_{1}\right]$ we can apply the Euclidean algorithm and get $p(\underline{z})=q_{1}\left(z_{1}\right) u_{1}(\underline{z})+v_{1}(\underline{z})$ where $v_{1}(\underline{z}) \in \mathbb{C}\left[z_{1}, \ldots, z_{n}\right]$ has a $z_{1}$-degree less than $\operatorname{deg} q_{1}$. Now we apply the Euclidean algorithm to $q_{2}\left(z_{2}\right)$ and $v_{1}$ as a polynomial in the variable $z_{2}$. Continuing this way we obtain

$$
s(\underline{z})-r(\underline{z})=\sum_{j=1}^{n} q_{j}\left(z_{j}\right) u_{j}(\underline{z})+v(\underline{z})
$$

By Lemma 6.1 we conclude $v=0$. Moreover, for $\underline{w} \in Z_{\underline{q}}^{\mathbb{R}}$ we have $\mathfrak{d}_{q_{j}}\left(w_{j}\right) \cdot e_{j} \in$ $I(\underline{w})$ and in turn

$$
0=D^{\mathfrak{d}_{q_{j}}\left(w_{j}\right) \cdot e_{j}}(s-r)(\underline{w})=q_{j}^{\left(\mathfrak{d}_{q_{j}}\left(w_{j}\right)\right)}\left(w_{j}\right) u_{j}(\underline{w}),
$$

where $q_{j}^{\left(\mathfrak{d}_{q_{j}}\left(w_{j}\right)\right)}\left(w_{j}\right) \neq 0$. Hence, $u_{j}(\underline{w})=0$ for all $j=1, \ldots, n$.

By (4.1) and [6, Lemma 5.11] we have

$$
\Xi_{j}\left(u_{j}\left(\Theta_{j}(\underline{A})\right)\right)=\Xi_{j}\left(\Theta_{j}\left(u_{j}(\underline{A})\right)\right)=q_{j}\left(A_{j}\right) u_{j}(\underline{A})
$$

for every $j \in\{1, \ldots, n\}$.

From $u_{j}\left(\Theta_{j}(\underline{A})\right)=\int u_{j} d E_{j},(3.9)$ and Corollary 4.3 we derive

$$
\begin{aligned}
\Xi_{j}\left(u_{j}\left(\Theta_{j}(\underline{A})\right)\right) & =\Xi_{j}\left(\int u_{j} d E_{j}\right)=\Xi\left(R_{j} R_{j}^{*} \int u_{j} d E\right) \\
& =\Xi\left(R_{j} R_{j}^{*} \int_{Z_{\underline{\mathbb{R}}}^{\mathbb{R}}} u_{j} d E+\int_{\sigma(\Theta(\underline{A})) \backslash Z_{\underline{q}}^{\mathbb{R}}} \frac{q_{j}\left(z_{j}\right) u_{j}(\underline{z})}{\sum_{k=1}^{n} q_{k}\left(z_{k}\right)} d E(\underline{z})\right) .
\end{aligned}
$$

Employing $(6.1),(6.2)$ and the fact that $u_{j}(\underline{w})=0$ for $\underline{w} \in Z_{\underline{q}}^{\mathbb{R}}$ we obtain

$$
s(\underline{A})-r(\underline{A})=\Xi\left(\int_{\sigma(\Theta(\underline{A})) \backslash Z_{\underline{q}}^{\mathbb{R}}} \frac{\sum_{j=1}^{n} q_{j}\left(z_{j}\right) u_{j}(\underline{z})}{\sum_{j=1}^{n} q_{j}\left(z_{j}\right)} d E(\underline{z})\right)
$$

On the other hand, since both $s, g$ and $r, h$ are decompositions of $\phi$ in sense of Lemma 5.19, we have

$$
\left(s_{\mathcal{M}}-r_{\mathcal{M}}\right)(\underline{z})=\sum_{j=1}^{n}(h(\underline{z})-g(\underline{z})) \cdot q_{j \mathcal{M}}\left(z_{j}\right)
$$


for $\underline{z} \in \sigma(\Theta(\underline{A}))$. In particular, for $\underline{z} \in \sigma(\Theta(\underline{A})) \backslash Z_{\underline{q}}^{\mathbb{R}}$

$$
\sum_{j=1}^{n} q_{j}\left(z_{j}\right) u_{j}(\underline{z})=(h(\underline{z})-g(\underline{z})) \cdot \sum_{j=1}^{n} q_{j}\left(z_{j}\right) .
$$

Since $h$ and $g$ vanish on $Z_{\underline{q}}^{\mathbb{R}}$, we obtain

$$
\begin{aligned}
s(\underline{A})-r(\underline{A}) & =\Xi\left(\int_{\sigma(\Theta(\underline{A})) \backslash Z_{\underline{\mathbb{R}}}^{\mathbb{R}}}(h(\underline{z})-g(\underline{z})) d E(\underline{z})\right) \\
& =\Xi\left(\int_{\sigma(\Theta(\underline{A}))}(h(\underline{z})-g(\underline{z})) d E(\underline{z})\right) .
\end{aligned}
$$

According to Lemma 6.2 the following definition does not depend on the actual choice of the decomposition of $\phi$.

Definition 6.3. If $\phi \in \mathcal{F}$ and if $s, g$ is an admissible decomposition of $\phi$ in the sense of Lemma 5.19, then we define

$$
\phi(\underline{A}):=s(\underline{A})+\Xi\left(\int_{\sigma(\Theta(\underline{A}))} g d E\right) .
$$

Theorem 6.4. The mapping $\phi \mapsto \phi(\underline{A})$ constitutes a *-homomorphism from $\mathcal{F}$ into $\underline{A}^{\prime \prime}\left(\subseteq L_{b}(\mathcal{K})\right)$ which satisfies $s_{\mathcal{M}}(\underline{A})=s(\underline{A})$ for every polynomial $s \in \mathbb{C}\left[z_{1}, \ldots, z_{n}\right]$.

Proof. Since $s_{\mathcal{M}}=s_{\mathcal{M}}+0 \cdot\left(\sum_{j=1}^{n} q_{j \mathcal{M}}(\underline{z})\right)$ is an admissible decomposition of $s_{\mathcal{M}}, s_{\mathcal{M}}(\underline{A})=s(\underline{A})$ is an immediate consequence of Definition 6.3.

Let $\phi_{1}, \phi_{2} \in \mathcal{F}$ and chose admissible decompositions $s_{1}, g_{1}$ of $\phi_{1}$ and $s_{2}, g_{2}$ of $\phi_{2}$ as in Lemma 5.19. Given $\lambda, \mu \in \mathbb{C}$, it is easily checked that $\lambda s_{1}+\mu s_{2}, \lambda g_{1}+\mu g_{2}$ is an admissible decomposition of $\lambda \phi_{1}+\mu \phi_{2}$. Therefore, the linearity $\Xi$ yields

$$
\begin{aligned}
\left(\lambda \phi_{1}+\mu \phi_{2}\right)(\underline{A})= & \left(\lambda s_{1}+\mu s_{2}\right)(\underline{A})+\Xi\left(\int_{\sigma(\Theta(\underline{A}))}\left(\lambda g_{1}+\mu g_{2}\right) d E\right) \\
= & \lambda\left(s_{1}(\underline{A})+\Xi\left(\int_{\sigma(\Theta(\underline{A}))} g_{1} d E\right)\right) \\
& +\mu\left(s_{2}(\underline{A})+\Xi\left(\int_{\sigma(\Theta(\underline{A}))} g_{2} d E\right)\right) \\
= & \lambda \phi_{1}(\underline{A})+\mu \phi_{2}(\underline{A}) .
\end{aligned}
$$

Obviously $s_{1}^{\#}, \overline{g_{1}}$ is an admissible decomposition for $\phi_{1}^{\#} \in \mathcal{F}$. From $\Xi\left(D^{*}\right)=$ $\Xi(D)^{+}$we derive

$$
\begin{aligned}
\phi_{1}(\underline{A})^{+} & =s_{1}(\underline{A})^{+}+\Xi\left(\int_{\sigma(\Theta(\underline{A}))} g_{1} d E\right)^{+} \\
& =s_{1}^{\#}(\underline{A})+\Xi\left(\int_{\sigma(\Theta(\underline{A}))} \overline{g_{1}} d E\right)=\phi_{1}^{\#}(\underline{A}) .
\end{aligned}
$$


Finally, we have

$$
\begin{aligned}
\phi_{1}(\underline{z}) \cdot \phi_{2}(\underline{z})= & \prod_{k=1,2}\left(\left(s_{k}\right)_{\mathcal{M}}(\underline{z})+g_{k}(\underline{z}) \cdot \sum_{j=1}^{n} q_{j}(\underline{z})\right) \\
= & \left(s_{1} s_{2}\right) \mathcal{M}(\underline{z}) \\
& +\left(s_{1}(\underline{z}) g_{2}(\underline{z})+s_{2}(\underline{z}) g_{1}(\underline{z})+g_{1}(\underline{z}) g_{2}(\underline{z}) \sum_{j=1}^{n} q_{j}(\underline{z})\right) \cdot \sum_{j=1}^{n} q_{j}(\underline{z})
\end{aligned}
$$

for all $\underline{z} \in \sigma(\Theta(\underline{A})) \cup Z_{\underline{q}}$. Since

$$
\sigma(\Theta(\underline{A})) \backslash Z_{\underline{q}}^{\mathbb{R}} \ni \underline{z} \mapsto s_{1}(\underline{z}) g_{2}(\underline{z})+s_{2}(\underline{z}) g_{1}(\underline{z})+g_{1}(\underline{z}) g_{2}(\underline{z}) \sum_{j=1}^{n} q_{j}(\underline{z}) \in \mathbb{C}
$$

is bounded, measurable and vanishes on $Z_{\underline{q}}, s_{1} s_{2}, s_{1} g_{2}+s_{2} g_{1}+g_{1} g_{2} \sum_{j=1}^{n} q_{j}$ is an admissible decomposition in the sense of Lemma 5.19 for $\phi_{1} \phi_{2} \in \mathcal{F}$; see Remark 5.15. Hence,

$$
\left(\phi_{1} \cdot \phi_{2}\right)(\underline{A})=\left(s_{1} \cdot s_{2}\right)(\underline{A})+\Xi\left(\int_{\sigma(\Theta(\underline{A}))}\left(s_{1} g_{2}+s_{2} g_{1}+g_{1} g_{2} \sum_{j=1}^{n} q_{j}\right) d E\right)
$$

Since by [6, Lemma 5.11] and (4.2) we have $\Xi\left(D_{1} D_{2} T^{+} T\right)=\Xi\left(D_{1}\right) \Xi\left(D_{2}\right)$, $\Xi(\Theta(C) D)=C \Xi(D), \Xi(D \Theta(C))=\Xi(D) C$ with $T^{+} T=\sum_{j=1}^{n} q_{j}\left(\Theta\left(A_{j}\right)\right)$, the second addend on the right hand side equals to

$$
\begin{aligned}
& \Xi\left(s_{1}(\Theta(\underline{A})) \int_{\sigma(\Theta(\underline{A}))} g_{2} d E+s_{2}(\Theta(\underline{A})) \int_{\sigma(\Theta(\underline{A}))} g_{1} d E\right. \\
& \left.+\left(\int_{\sigma(\Theta(\underline{A}))} g_{1} g_{2} d E\right) \sum_{j=1}^{n} q_{j}\left(\Theta\left(A_{j}\right)\right)\right) \\
& =s_{1}(\underline{A}) \Xi\left(\int_{\sigma(\Theta(\underline{A}))} g_{2} d E\right)+\Xi\left(\int_{\sigma(\Theta(\underline{A}))} g_{1} d E\right) s_{2}(\underline{A}) \\
& \quad+\Xi\left(\int_{\sigma(\Theta(\underline{A}))} g_{1} d E\right) \Xi\left(\int_{\sigma(\Theta(\underline{A}))} g_{2} d E\right)
\end{aligned}
$$

Therefore,

$$
\begin{aligned}
& \left(\phi_{1} \cdot \phi_{2}\right)(\underline{A})=\left(s_{1}(\underline{A})+\Xi\left(\int_{\sigma(\Theta(\underline{A}))} g_{1} d E\right)\right) \\
& \quad\left(s_{2}(\underline{A})+\Xi\left(\int_{\sigma(\Theta(\underline{A}))} g_{2} d E\right)\right) \\
& =\phi_{1}(\underline{A}) \phi_{2}(\underline{A})
\end{aligned}
$$

Finally, we shall show that $\phi(\underline{A}) \in \underline{A}^{\prime \prime}$. Clearly, given an admissible decomposition $s, g$ with $s \in \mathbb{C}\left[z_{1}, \ldots, z_{n}\right]$ we have $s(\underline{A}) \in \underline{A}^{\prime \prime}$. If $C \in \underline{A}^{\prime} \subseteq$ $\bigcap_{j=1}^{n}\left(T_{j} T_{j}^{+}\right)^{\prime}$, then $\Theta(C) \in\{\Theta(\underline{A})\}^{\prime}$ because $\Theta$ is a homomorphism. By the spectral theorem in Hilbert spaces $\Theta(C)$ commutes with $E(\Delta)$ for all Borel sets $\Delta$. Consequently, it commutes with 


$$
D:=\int_{\sigma(\Theta(\underline{A}))} g d E .
$$

From [6, Lemma 5.11] we infer $\Xi(D) C=\Xi(D \Theta(C))=\Xi(\Theta(C) D)=C \Xi(D)$. Hence, $\Xi(D) \in \underline{A}^{\prime \prime}$ and finally $\phi(\underline{A}) \in \underline{A}^{\prime \prime}$.

Proposition 6.5. The functional calculus $\phi \mapsto \phi\left(A_{1}, \ldots, A_{n}\right)$ in Theorem 6.4 is bounded, when $\mathcal{F}$ is provided with \|\|$_{\mathcal{F}}$ and $L_{b}(\mathcal{K})$ is provided with the operator norm which originates from some compatible Hilbert space scalar product on $\mathcal{K}$.

Proof. The mapping $\mathcal{F} \ni \phi \mapsto s \in \mathbb{C}\left[z_{1}, \ldots, z_{n}\right]$ from Lemma 5.10 is linear. Thereby, the coefficients of $s$ depend continuously on $\phi$; see Remark 5.11. Consequently, also $s(\underline{A})$ depends continuously on $\phi$.

By Lemma 5.20 the bounded $g \in \mathcal{B}\left(\sigma(\Theta(\underline{A})) \cup Z_{\underline{q}}^{\mathbb{R}}, \mathbb{C}\right)$, such that $s, g$ is an admissible decomposition of $\phi$, depends continuously on $\phi$. Thus, $\int g d E$, and in turn $\Xi\left(\int g d E\right)$, depend continuously on $\phi$.

\section{Spectrum of $\underline{A}$}

As in the previous section let $\underline{A} \in L_{b}(\mathcal{K})^{n}$ be a tuple of pairwise commuting, bounded, self-adjoint and definitizable operators where for $j=1, \ldots, n$ we denote by $q_{j} \in \mathbb{R}[\zeta] \backslash\{0\}$ a definitizing polynomial for $A_{j}$. We shall employ the same notation as the previous sections. The aim of the present section is to describe the spectrum $\sigma(\underline{A})\left(\subseteq \mathbb{C}^{n}\right)$ of the tuple $\underline{A}$; see Definition 2.1.

Remark 7.1. If $\underline{w} \notin \sigma(\underline{A})$, then $(\underline{A}-\underline{w}) \cdot \underline{B}=\sum_{j=1}^{n}\left(A_{j}-w_{j}\right) B_{j}=I$ for some $\underline{B}=\left(B_{j}\right)_{j=1}^{n} \in\left(\underline{A}^{\prime \prime}\right)^{n} \subseteq L_{b}(\mathcal{K})^{n}$. Taking adjoints and using the fact that $\underline{A}^{\prime \prime}$ is abelian yields $\sum_{j=1}^{n}\left(A_{j}-\bar{w}_{j}\right)^{+} B_{j}^{+}=I$ which means $\underline{\bar{w}} \notin \sigma(\underline{A})$. Hence,

$$
\overline{\sigma(\underline{A})}=\sigma(\underline{A}) \text {. }
$$

Since $\Theta:\left(T T^{+}\right)^{\prime} \rightarrow\left(T^{+} T\right)^{\prime}$ constitutes a $*$-homomorphism, we also have

$$
\sigma(\Theta(\underline{A})) \subseteq \sigma(\underline{A}) \text {. }
$$

Remark 7.2. Choosing $s_{j} \in \mathbb{C}\left[z_{1}, \ldots, z_{n}\right]$ with $s_{j}\left(z_{1}, \ldots, z_{n}\right)=z_{j}$ we obtain from Theorem $6.4\left(s_{j}\right)_{\mathcal{M}}(\underline{A})=A_{j}$.

Let $\underline{w} \in Z_{\underline{q}}$ be an isolated point of $\sigma(\Theta(\underline{A})) \cup Z_{\underline{q}}$ and let $e$ be the multiplicative neutral element of $\mathbb{C}^{I(\underline{w})}$; see Remark 5.3. By Example 5.16 the function $\delta_{\underline{w}} e$ belongs to $\mathcal{F}$. As $\delta_{\underline{w}} e \cdot \delta_{\underline{w}} e=\delta_{\underline{w}} e$ the corresponding operator $P_{\underline{w}}:=\left(\delta_{\underline{w}} e\right)(\underline{A}) \in \underline{A}^{\prime \prime} \subseteq L_{b}(\mathcal{K})$ constitutes a projection. Since $P_{\underline{w}}$ commutes with all operators of the form $\phi(\underline{A})$ where $\phi \in \mathcal{F}$, the range of $P_{\underline{w}}$ is invariant under all these operators $\phi(\underline{A})$, in particular under $A_{j}$ for $j=\overline{1}, \ldots, n$.

For $\underline{\lambda} \in \mathbb{C}^{n} \backslash\{\underline{w}\}$ we have $\left(s_{j}(\underline{w})-\lambda_{j}\right)=w_{j}-\lambda_{j} \neq 0$ for at least one $j \in\{1, \ldots, n\}$. According to Remark $5.3\left(s_{j}-\lambda_{j}\right)_{\mathcal{M}}(\underline{w}) \in \mathbb{C}^{I(\underline{w})}$ is then invertible with an inverse $b_{j} \in \mathbb{C}^{I(\underline{w})}$. From

$$
\left(s_{j}-\lambda_{j}\right)_{\mathcal{M}} \cdot\left(\delta_{\underline{w}} e\right)=\delta_{\underline{w}}\left(\left(s_{j}-\lambda_{j}\right)_{\mathcal{M}}(\underline{w})\right)(\subseteq \mathcal{F})
$$


we derive for $x=P_{\underline{w}} x \in \operatorname{ran} P_{\underline{w}}$

$$
\begin{array}{r}
\left(s_{j}-\lambda_{j}\right)_{\mathcal{M}}(\underline{A}) \cdot\left(\delta_{\underline{w}} b_{j}\right)(\underline{A}) x \\
=\left(\delta_{\underline{w}}\left(\left(s_{j}-\lambda_{j}\right)_{\mathcal{M}}(\underline{w}) \cdot b_{j}\right)\right)(\underline{A}) x=x .
\end{array}
$$

and conclude that

$$
\left.\left(A_{j}-\lambda_{j}\right)\right|_{\operatorname{ran} P_{\underline{w}}}=\left.A_{j}\right|_{\operatorname{ran} P_{\underline{w}}}-\left.\lambda_{j} I\right|_{\operatorname{ran} P_{\underline{w}}}
$$

has $\left.\left(\delta_{\underline{w}} b_{j}\right)(\underline{A})\right|_{\text {ran } P_{\underline{w}}}$ as its inverse operator. From (2.2) we obtain $\underline{\lambda} \notin \sigma\left(\left(\left.A_{j}\right|_{\operatorname{ran} P_{\underline{w}}}\right)_{j=1}^{n}\right)$ and in turn $\sigma\left(\left(\left.A_{j}\right|_{\operatorname{ran} P_{\underline{w}}}\right)_{j=1}^{n}\right) \subseteq\{\underline{w}\} . \diamond$

Lemma 7.3. For any point $\underline{w} \in Z_{\underline{q}} \backslash \sigma(\underline{A})$ we have $\left(\delta_{\underline{w}} e\right)(\underline{A})=0$.

Proof. By Remark 7.1 the point $\underline{w} \in Z_{\underline{q}} \backslash \sigma(\underline{A})$ is isolated in $\sigma(\Theta(\underline{A})) \cup Z_{\underline{q}}$. Hence, by Remark 7.2 the projection $P_{\underline{w}}:=\left(\delta_{\underline{w}} e\right)(\underline{A}) \in \underline{A}^{\prime \prime}$ is well defined.

By assumption the operator tuple $\underline{A}-\underline{w}\left(\in L_{b}(\mathcal{K})^{n}\right)$ is invertible which means that $\sum_{j=1}^{n}\left(A_{j}-w_{j} I\right) B_{j}=I$ for some $B_{1}, \ldots, B_{n} \in \underline{A}^{\prime \prime}$; see Definition 2.1. Since $P_{w}$ and $B_{1}, \ldots, B_{n}$ belong to a commutative subalgebra of $L_{b}(\mathcal{K})$, we have $\bar{B}_{1}\left(\operatorname{ran} P_{\underline{w}}\right), \ldots, B_{n}\left(\operatorname{ran} P_{\underline{w}}\right) \subseteq \operatorname{ran} P_{\underline{w}}$. This yields

$$
\left.\sum_{j=1}^{n}\left(\left.A_{j}\right|_{\operatorname{ran} P_{\underline{w}}}-\left.w_{j} I\right|_{\operatorname{ran} P_{\underline{w}}}\right) B_{j}\right|_{\operatorname{ran} P_{\underline{w}}}=\left.I\right|_{\operatorname{ran} P_{\underline{w}}},
$$

i.e. $w \notin \sigma\left(\left(\left.A_{j}\right|_{\operatorname{ran} P_{\underline{w}}}\right)_{j=1}^{n}\right)$. According to Remark $7.2 \sigma\left(\left(\left.A_{j}\right|_{\operatorname{ran} P_{\underline{w}}}\right)_{j=1}^{n}\right)=\emptyset$, which is only possible if $\operatorname{ran} P_{w}=0$ or equivalently $\left(\delta_{\underline{w}} e\right)(\underline{A})=0$.

Corollary 7.4. The spectrum of $\underline{A}=\left(A_{j}\right)_{j=1}^{n}$ satisfies

$$
\sigma(\underline{A})=\sigma(\Theta(\underline{A})) \cup\left(\sigma(\underline{A}) \cap Z_{\underline{q}}\right) .
$$

Proof. By Remark 7.1 it is enough to show that for $\underline{\lambda} \in \mathbb{C}^{n} \backslash(\sigma(\Theta(\underline{A})) \cup$ $\left.\left(\sigma(\underline{A}) \cap Z_{q}\right)\right)$ the operator tuple $\underline{A}-\underline{\lambda}$ is invertible.

For every $\underline{w} \in \sigma(\Theta(\underline{A})) \cup\left(\sigma(\underline{A}) \cap Z_{\underline{q}}\right)$ let $c \underline{w}, \ldots, c \underline{w} \in \mathbb{C}$ be such that

$$
\sum_{j=1}^{n}\left(w_{j}-\lambda_{j}\right)\left(w_{j}-\bar{\lambda}_{j}+c_{j}^{\frac{w}{j}}\right) \neq 0 \text {. }
$$

Such a choice is possible because $\underline{\lambda} \notin \sigma(\Theta(\underline{A})) \cup\left(\sigma(\underline{A}) \cap Z_{q}\right)$ and hence $w_{j}-\lambda_{j} \neq 0$ for some $j$. For $s_{j}$ as in Remark 7.2 the functions

$$
\phi_{j}:=\left(s_{j}-\bar{\lambda}_{j}\right)_{\mathcal{M}}+\sum_{\underline{w} \in \sigma(\Theta(\underline{A})) \cup\left(\sigma(\underline{A}) \cap Z_{\underline{q}}\right)} c_{j}^{\frac{w}{j}}\left(\delta_{\underline{w}} e\right) \in \mathcal{F}, \quad j=1, \ldots, n,
$$

satisfies $\left(\phi_{j}(\underline{w})\right)_{(0, \ldots, 0)}=\left(w_{j}-\bar{\lambda}_{j}+c c_{j}^{w}\right)$ for $\underline{w} \in \sigma(\Theta(\underline{A})) \cup\left(\sigma(\underline{A}) \cap Z_{\underline{q}}\right)$. With $d^{\underline{w}}=1-\sum_{j=1}^{n}\left(w_{j}-\lambda_{j}\right)\left(w_{j}-\bar{\lambda}_{j}\right), \underline{w} \in Z_{\underline{q}} \backslash \sigma(\underline{A})$ consider

$$
\phi:=\sum_{j=1}^{n}\left(s_{j}-\lambda_{j}\right)_{\mathcal{M}} \cdot \phi_{j}+\sum_{\underline{w} \in Z_{\underline{q}} \backslash \sigma(\underline{A})} d^{\underline{w}}\left(\delta_{\underline{w}} e\right) .
$$

We have $(\phi(\underline{w}))_{(0, \ldots, 0)}=\sum_{j=1}^{n}\left(w_{j}-\lambda_{j}\right)\left(w_{j}-\bar{\lambda}_{j}+c c_{j}^{w}\right) \neq 0$ for $\underline{w} \in$ $\sigma(\Theta(\underline{A})) \cup\left(\sigma(\underline{A}) \cap Z_{\underline{q}}\right)$ and $(\phi(\underline{w}))_{(0, \ldots, 0)}=1$ for $\underline{w} \in Z_{\underline{q}} \backslash \sigma(\underline{A})$. Hence, $\phi(\underline{w}) \in$ 
$\mathbb{C}^{I(\underline{w})}$ is invertible for all $\underline{w} \in Z_{q} \backslash \sigma(\Theta(\underline{A}))$. For $z \in \sigma(\Theta(\underline{A})) \backslash Z_{q} \subseteq \mathbb{R}^{n}$ we have

$$
\begin{aligned}
(\phi(\underline{z}))_{(0, \ldots, 0)} & =\sum_{j=1}^{n}\left(s_{j}(\underline{z})-\lambda_{j}\right) \cdot\left(s_{j}(\underline{z})-\bar{\lambda}_{j}\right) \\
& =\|\underline{z}-\underline{\lambda}\|_{2}^{2} \geq d(\underline{\lambda}, \sigma(\Theta(\underline{A})))>0 .
\end{aligned}
$$

We see that all assumptions of Lemma 5.18 are satisfied. Hence, $\phi^{-1} \in$ $\mathcal{F}$. If we set $B_{j}=\left(\phi^{-1} \cdot \phi_{j}\right)(\underline{A})$ for $j=1, \ldots, n$, then we obtain

$$
\sum_{j=1}^{n}\left(A_{j}-\lambda_{j} I\right) B_{j}=\left(\phi^{-1} \cdot \sum_{j=1}^{n}\left(s_{j}-\lambda_{j}\right)_{\mathcal{M}} \cdot \phi_{j}\right)(\underline{A}) .
$$

By Lemma 7.3 this expression coincides with

$$
\left(\phi^{-1} \sum_{j=1}^{n}\left(s_{j}-\lambda_{j}\right)_{\mathcal{M}} \cdot \phi_{j}+\sum_{\underline{w} \in Z_{\underline{q}} \backslash \sigma(\underline{A})} d^{\underline{w}}\left(\delta_{\underline{w}} e\right)\right)(\underline{A})=(\mathbb{1})_{\mathcal{M}}(\underline{A})=I .
$$

Thus, $\underline{A}-\underline{\lambda}$ is invertible.

Lemma 7.5. Let $\phi \in \mathcal{F}$. If $\phi(\underline{z})=0$ for all $\underline{z} \in \sigma(\underline{A})$, then $\phi(\underline{A})=0$.

Proof. Our assumptions together with Corollary 7.4 implies that $\phi$ can be written as $\sum_{\underline{w} \in Z_{\underline{q}} \backslash \sigma(\underline{A})} \delta_{\underline{w}} \phi(\underline{w})$. By Lemma 7.3 we obtain

$$
\begin{aligned}
& \phi(\underline{A})=\sum_{\underline{w} \in Z_{\underline{q}} \backslash \sigma(\underline{A})}\left(\delta_{\underline{w}} \phi(\underline{w})\right)(\underline{A}) \\
& =\sum_{\underline{w} \in Z_{\underline{q}} \backslash \sigma(\underline{A})}\left(\delta_{\underline{w}} \phi(\underline{w})\right)(\underline{A})\left(\delta_{\underline{w}} e\right)(\underline{A})=0 .
\end{aligned}
$$

Remark 7.6. The previous result implies for $\phi \in \mathcal{F}$ that $\phi(\underline{A})$ only depends $\phi(\underline{z})$, where $\underline{z}$ runs in $\sigma(\underline{A})$. Indeed, if $\phi_{1}(\underline{z})=\phi_{2}(\underline{z})$ for all $\underline{z} \in \sigma(\underline{A})$, then by Lemma 7.5 we obtain $\phi_{1}(\underline{A})-\phi_{2}(\underline{A})=0$ and hence $\phi_{1}(\underline{A})=\phi_{2}(\underline{A})$. $\diamond$

Since we can alter the values of a function $\phi \in \mathcal{F}$ at all point in $Z_{\underline{q}} \backslash \sigma(\underline{A})$ without changing $\phi(\underline{A})$, we derive from Lemma 5.18 the following result.

Lemma 7.7. If $\phi \in \mathcal{F}$ is such that $\phi(\underline{z})$ is invertible in $\mathbb{C}^{I(\underline{z})}$ for all $z \in \sigma(\underline{A})$ and such that 0 does not belong to the closure of $\phi\left(\sigma(\Theta(\underline{A})) \backslash Z_{q}^{\mathbb{R}}\right)$, then $\phi(\underline{A})$ is invertible. Its inverse coincides with $\psi(\underline{A})$ for any $\psi \in \mathcal{F}$ satisfying $\psi(\underline{z})=$ $\phi(\underline{z})^{-1}, \underline{z} \in \sigma(\underline{A})$.

\section{Normal Operators}

In [4] normal operators $N \in L_{b}(\mathcal{K})$ on a Krein space $\mathcal{K}$, which are definitizable in the sense that their real part $A_{1}:=\frac{1}{2}\left(N+N^{+}\right)$and their imaginary part $A_{2}:=\frac{1}{2 \mathrm{i}}\left(N-N^{+}\right)$are definitizable, were considered. The results derived in that work perfectly fit into our present framework. 
Indeed, for a normal definitizable $N \in L_{b}(\mathcal{K})$ the pair $A_{1}, A_{2} \in L_{b}(\mathcal{K})$ constitutes a tuple as considered in Sect. 4 . The following result describes the connection of the spectrum of $N$ and the spectrum of the tuple $\left(A_{1}, A_{2}\right)$.

Lemma 8.1. Let $N$ be normal and definitizable operator in a Krein space $\mathcal{K}$ and $A_{1}, A_{2}$ the corresponding real and imaginary part of $N$. Then we have

$$
\sigma(N)=\left\{z_{1}+\mathrm{i} z_{2}: \underline{z} \in \sigma\left(\left(A_{1}, A_{2}\right)\right)\right\} .
$$

Proof. If $\eta \in \mathbb{C} \backslash \sigma(N)$, then $(N-\eta)^{-1}$ exists as an element of $L_{b}(\mathcal{K})$. We set $B_{1}:=(N-\eta)^{-1}$ and $B_{2}:=\mathrm{i}(N-\eta)^{-1}$. Clearly, $B_{1}, B_{2} \in\left\{A_{1}, A_{2}\right\}^{\prime \prime}$. For every $\underline{\lambda} \in \mathbb{C}^{2}$ which fulfills $\lambda_{1}+\mathrm{i} \lambda_{2}=\eta$ we have

$$
\left(A_{1}-\lambda_{1}\right) B_{1}+\left(A_{2}-\lambda_{2}\right) B_{2}=(\underbrace{A_{1}+\mathrm{i} A_{2}}_{=N}-\underbrace{\left(\lambda_{1}+\mathrm{i} \lambda_{2}\right)}_{=\eta})(N-\eta)^{-1}=I .
$$

Thus, $\left(A_{1}-\lambda_{1}, A_{2}-\lambda_{2}\right)$ is invertible which means that $\underline{\lambda} \in \mathbb{C}^{2} \backslash \sigma\left(\left(A_{1}, A_{2}\right)\right)$.

Conversely, for $\eta \in \mathbb{C} \backslash\left\{z_{1}+\mathrm{i} z_{2}: \underline{z} \in \sigma\left(\left(A_{1}, A_{2}\right)\right)\right\}$ the function $f_{\mathcal{M}}$, where $f: \mathbb{C}^{2} \rightarrow \mathbb{C}$ is defined by $f(\underline{z}):=z_{1}+\mathrm{i} z_{2}-\eta$, satisfies the conditions of Lemma 7.7. Therefore, $f_{\mathcal{M}}$ has a multiplicative inverse. From $f_{\mathcal{M}}\left(A_{1}, A_{2}\right)=$ $N-\eta$ we finally conclude $\eta \in \mathbb{C} \backslash \sigma(N)$.

The functional calculus developed in [4] for normal $N=A_{1}+\mathrm{i} A_{2}$, definitizable operators on Krein spaces is almost the same as the functional calculus for the tuple $A_{1}, A_{2}$ from the present paper. The only difference is the domain for the functions $\phi \in \mathcal{F}$. In the present note $\phi$ is defined on the compact subset

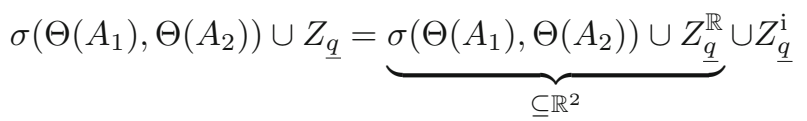

of $\mathbb{C}^{2}$ whereas in [4] $\phi$ is defined on

$$
\underbrace{\sigma(\Theta(N)) \cup\left\{z_{1}+\mathrm{i} z_{2}: \underline{z} \in Z_{\underline{q}}^{\mathbb{R}}\right\}}_{\subseteq \mathbb{C}} \cup \underbrace{Z_{\underline{q}}^{\mathrm{i}}}_{\subseteq \mathbb{C}^{2}},
$$

where according to Lemma 8.1 the spectrum of the normal operator $\Theta(N)=$ $\Theta\left(A_{1}\right)+\mathrm{i} \Theta\left(A_{2}\right)$ on the Hilbert space $\mathcal{H}$ coincides with $\left\{z_{1}+\mathrm{i} z_{2}: \underline{z} \in\right.$ $\left.\sigma\left(\left(\Theta\left(A_{1}\right), \Theta\left(A_{2}\right)\right)\right)\right\}$. Since $\mathbb{R}^{2} \ni \underline{z} \mapsto z_{1}+\mathrm{i} z_{2} \in \mathbb{C}$ is bijective, the sets in (8.1) and (8.2) correspond to each other.

\section{Compatibility of the Spectral Theorem}

In this section we want to regard the spectral calculus for a tuple $\underline{A}_{N}=$ $\left(A_{j}\right)_{j=1}^{n}$ compared to the spectral calculus for $\underline{A}_{M}:=\left(A_{j}\right)_{j \in M}$, where $M \subseteq$ $N=\{1, \ldots, n\}$ has $m$ elements. For this we again fix definitizing polynomials $q_{j} \in \mathbb{R}[\zeta] \backslash\{0\}$ for $A_{j}, j=1, \ldots, n$, and set $\underline{q}_{N}=\left(q_{j}\right)_{j=1}^{n}$ and $\underline{q}_{M}=\left(q_{j}\right)_{j \in M}$. We will employ the notation used in Sect. $\overline{4}$.

Moreover, we shall denote the function class introduced in Definition 5.13, which corresponds to $\underline{A}_{M}$, by $\mathcal{M}_{M}$ and $\mathcal{F}_{M}$, and the function class, which 
corresponds to $\underline{A}_{N}$, by $\mathcal{M}_{N}$ and $\mathcal{F}_{N}$. Finally, we introduce the projection $\pi: \mathbb{C}^{N} \rightarrow \mathbb{C}^{M}$ defined by

$$
\pi(\underline{z}):=\left(z_{j}\right)_{j \in M} .
$$

Note that by Theorems 2.5, 2.4 and (3.4)

$$
\pi\left(\sigma\left(\Theta_{N}\left(\underline{A}_{N}\right)\right)\right)=\sigma\left(\Theta_{N}\left(\underline{A}_{M}\right)\right) \supseteq \sigma\left(\Theta_{M}\left(\underline{A}_{M}\right)\right) .
$$

As $Z_{\underline{q}_{N}}=\prod_{j=1}^{n} Z_{q_{j}}$ and $Z_{\underline{q}_{M}}=\prod_{j \in M} Z_{q_{j}}$ we also have $\pi\left(Z_{\underline{q}_{N}}\right)=Z_{\underline{q}_{M}}$. According to (2.3) we have $\pi\left(\sigma\left(\underline{A}_{N}\right)\right) \subseteq \sigma\left(\underline{A}_{M}\right)$ which by Corollary 7.4 implies

$$
\pi\left(\sigma\left(\Theta_{N}\left(\underline{A}_{N}\right)\right) \cup Z_{\underline{q}_{N}}\right)=\sigma\left(\Theta_{M}\left(\underline{A}_{M}\right)\right) \cup Z_{\underline{q}_{M}} .
$$

Definition 9.1. For $\phi \in \mathcal{M}_{M}$ we define $\phi \circ \pi \in \mathcal{M}_{N}$ by

$$
(\phi \circ \pi(\underline{z}))_{\alpha}= \begin{cases}\phi(\pi(\underline{z}))_{\pi(\alpha)}, & \text { if } \alpha_{j}=0 \text { for all } j \in N \backslash M \\ 0, & \text { otherwise }\end{cases}
$$

for $\alpha \in I(\underline{z})$ and $\underline{z} \in \sigma\left(\Theta_{N}\left(\underline{A}_{N}\right)\right) \cup Z_{\underline{q}_{N}}$. Here $I(\underline{z}) \subseteq \mathbb{Z}^{N}$ is defined as in (5.2) on the base of tuple $\underline{A}_{N} \cdot \diamond$

For $\underline{z} \in \sigma\left(\Theta_{N}\left(\underline{A}_{N}\right)\right) \cup Z_{\underline{q}_{N}}$ we conclude from $\underline{z} \in Z_{\underline{q}_{N}}^{\mathbb{R}}$ that $\pi(\underline{z}) \in Z_{\underline{q}_{M}}^{\mathbb{R}}$ and $\pi(\alpha) \in\left[0, \mathfrak{d}_{\underline{q}_{M}}(\pi(\underline{z}))\right]$ for all $\alpha \in\left[0, \mathfrak{d}_{\underline{q}_{N}}(\underline{z})\right]$ and from $\underline{z} \in Z_{\underline{q}_{N}}^{\mathrm{i}}$ that $\pi(\underline{z}) \in Z_{\underline{q}_{M}}$ and $\pi(\alpha) \in\left[0, \mathfrak{d}_{\underline{q}_{M}}(\pi(\underline{z}))\right)$ for all $\alpha \in\left[0, \mathfrak{d}_{\underline{q}_{N}}(\underline{z})\right)$. Thus, $\phi \circ \pi$ as defined in (9.1) belongs to $\mathcal{M}_{N}$.

Lemma 9.2. For every $\phi \in \mathcal{F}_{M}$ we have $\phi \circ \pi \in \mathcal{F}_{N}$.

Moreover, if for $s \in \mathbb{C}\left[z_{j}, j \in M\right]$ we denote by $s \circ \pi$ the polynomial $s$ as an element of $\mathbb{C}\left[z_{j}, j \in N\right]$, then $(s \circ \pi)_{\mathcal{M}_{N}}=s_{\mathcal{M}_{M}} \circ \pi$; see Definition 5.7 .

Proof. For $\underline{w} \in Z_{\underline{q}_{N}}$ such that $\underline{w}$ is not isolated in $\sigma\left(\Theta_{N}\left(\underline{A}_{N}\right)\right) \cup Z_{\underline{q}_{N}}$ and such that $\pi(\underline{w}) \in \underline{Z}_{\underline{q}_{M}}$ is not isolated in $\sigma\left(\Theta_{M}\left(\underline{A}_{M}\right)\right) \cup Z_{\underline{q}_{M}}$ we have

$$
\phi(\underline{\zeta})=\sum_{\beta \in\left[0, \mathfrak{d}_{\underline{q}_{M}}(\pi(\underline{w}))\right)}(\phi(\underline{w}))_{\beta}(\underline{\zeta}-\pi(\underline{w}))^{\beta}+O\left(\max _{j \in M}\left|\zeta_{j}-w_{j}\right|^{\mathfrak{d}_{q_{j}}\left(w_{j}\right)}\right)
$$

as $\sigma\left(\Theta_{M}\left(\underline{A}_{M}\right)\right) \backslash Z_{\underline{q}_{M}}^{\mathbb{R}} \ni \underline{\zeta} \rightarrow \pi(\underline{w})$. Substituting $\underline{\zeta}=\pi(\underline{z})$ with $\underline{z} \in \sigma\left(\Theta_{N}\left(\underline{A}_{N}\right)\right)$ $\backslash Z_{\underline{q}_{N}}^{\mathbb{R}}$ and employing (9.1), $(\phi \circ \pi(\underline{z}))_{\alpha}=0$ for all $\alpha$ not satisfying $\alpha_{j}=0, j \in$ $N \backslash M$, and the fact that $(\underline{z}-\underline{w})^{\alpha}=(\pi(\underline{z})-\pi(\underline{w}))^{\pi(\alpha)}$ for $\alpha_{j}=0, j \in N \backslash M$, yields

$$
\phi(\pi(\underline{z}))=\sum_{\alpha \in\left[0, \mathfrak{d}_{\underline{q}_{N}}(\underline{w})\right)}(\phi(w))_{\alpha}(\underline{z}-\underline{w})^{\alpha}+O\left(\max _{j \in M}\left|z_{j}-w_{j}\right|^{\mathfrak{d}_{q_{j}}\left(w_{j}\right)}\right)
$$

as $\sigma\left(\Theta_{N}\left(\underline{A}_{N}\right)\right) \backslash Z_{\underline{q}_{N}}^{\mathbb{R}} \ni \underline{z} \rightarrow \underline{w}$. From $\max _{j \in M}\left|z_{j}-w_{j}\right|^{\mathfrak{o}_{q_{j}}\left(w_{j}\right)}=O\left(\max _{j \in N} \mid z_{j}-\right.$ $\left.\left.w_{j}\right|^{\mathfrak{d}_{q_{j}}\left(w_{j}\right)}\right)$ we obtain $\phi \circ \pi \in \mathcal{F}_{N}$. 
If $\underline{w} \in Z_{\underline{q}_{N}}$ is such that $\underline{w}$ is not isolated in $\sigma\left(\Theta_{N}\left(\underline{A}_{N}\right)\right) \cup Z_{\underline{q}_{N}}$ and such that $\pi(\underline{w}) \in \underline{Z}_{\underline{q}_{M}}$ is isolated in $\sigma\left(\Theta_{M}\left(\underline{A}_{M}\right)\right) \cup Z_{\underline{q}_{M}}$, then $z_{j}-w_{j}=0$ for $j \in M$ and for $\underline{z} \in \sigma\left(\Theta_{N}\left(\underline{A}_{N}\right)\right) \backslash Z_{\underline{q}_{N}}^{\mathbb{R}}$ sufficiently close to $\underline{w}$. Hence,

$$
\phi(\pi(\underline{z}))=\phi(\pi(\underline{w}))=\sum_{\alpha \in\left[0, \mathfrak{o}_{\underline{q}_{N}}(\underline{w})\right)}(\phi(w))_{\alpha}(\underline{z}-\underline{w})^{\alpha}
$$

for $\underline{z} \in \sigma\left(\Theta_{N}\left(\underline{A}_{N}\right)\right) \backslash Z_{\underline{q}_{N}}^{\mathbb{R}}$ sufficiently close to $w$.

In order to verify the final assertion, it is obviously enough to show that $(s \circ \pi)_{\mathcal{M}_{N}}(\underline{z})=\left(s_{\mathcal{M}_{M}} \circ \pi\right)(\underline{z})$ for $z \in Z_{\underline{q}_{N}}$. In fact, we have

$$
\frac{1}{\alpha !} D^{\alpha} s \circ \pi(\underline{z})=0=\left(\left(s_{\mathcal{M}_{M}} \circ \pi\right)(\underline{z})\right)_{\alpha}
$$

if $\alpha_{j} \neq 0$ for some $j \in N \backslash M$ and

$$
\begin{aligned}
\frac{1}{\alpha !} D^{\alpha} s \circ \pi(\underline{z}) & =\frac{1}{\pi(\alpha)} D^{\pi(\alpha)} s(\pi(\underline{z}))=\left(s_{\mathcal{M}_{M}}(\pi(\underline{z}))\right)_{\pi(\alpha)} \\
& =\left(\left(s_{\mathcal{M}_{M}} \circ \pi\right)(\underline{z})\right)_{\alpha}
\end{aligned}
$$

if $\alpha_{j}=0$ for all $j \in N \backslash M$.

Remark 9.3. It is straight forward to verify that $\mathcal{F}_{M} \ni \phi \mapsto \phi \circ \pi(\underline{z}) \in \mathcal{F}_{N}$ is linear and respects multiplication on $\mathcal{F}$. $\diamond$

Corollary 9.4. If $s, g$ is an admissible decomposition of $\phi \in \mathcal{F}_{M}$ in the sense of Lemma 5.19 with $s \in \mathbb{C}\left[z_{j}, j \in M\right]$ and a measurable and bounded $g$ : $\sigma\left(\Theta_{M}\left(\underline{A}_{M}\right)\right) \cup Z_{\underline{q}_{M}} \rightarrow \mathbb{C}$, then $s \circ \pi \in \mathbb{C}\left[z_{j}, j \in N\right]$ and $h: \sigma\left(\Theta_{N}\left(\underline{A}_{N}\right)\right) \cup$ $Z_{\underline{q}_{N}} \rightarrow \mathbb{C}$ is an admissible decomposition of $\phi \circ \pi \in \mathcal{F}_{N}$ where

$$
h(\underline{z})=g(\pi(\underline{z})) \cdot \frac{\sum_{j \in M} q_{j}\left(z_{j}\right)}{\sum_{j \in N} q_{j}\left(z_{j}\right)}, \quad \underline{z} \in \sigma\left(\Theta_{N}\left(\underline{A}_{N}\right)\right) \cup Z_{\underline{q}_{N}} .
$$

Proof. By Lemma 5.19 we have

$$
\phi=s_{\mathcal{M}_{M}}+g \cdot\left(\sum_{j \in M}\left(q_{j}\right)_{\mathcal{M}_{M}}\right)
$$

with a bounded an measurable function $\left.g\right|_{\sigma\left(\Theta_{M}\left(\underline{A}_{M}\right)\right) \backslash Z_{\underline{q}_{M}}^{\mathrm{R}}}$. By Proposition 4.2 $\left.h\right|_{\sigma\left(\Theta_{N}\left(\underline{A}_{N}\right)\right) \backslash Z_{\underline{q}_{N}}^{\mathrm{R}}}$ is also bounded and measurable.

As $\left.g\right|_{Z_{\underline{q}_{M}}} \equiv 0$ we conclude from Remark 9.3 and Lemma 9.2 that $\phi \circ$ $\pi(\underline{w})=(s \circ \pi)_{\mathcal{M}_{N}}(\underline{w})$ and $h(\underline{w})=0$ for $\underline{w} \in Z_{\underline{q}_{N}}$. For $\underline{z} \in \sigma\left(\Theta_{N}\left(\underline{A}_{N}\right)\right) \backslash Z_{\underline{q}_{N}}^{\mathbb{R}}$ we have

$$
\begin{aligned}
\phi \circ \pi(\underline{z}) & =s_{\mathcal{M}_{M}} \circ \pi(\underline{z})+g(\pi(\underline{z})) \cdot\left(\sum_{j \in M}\left(q_{j}\right)_{\mathcal{M}_{M}} \circ \pi(\underline{z})\right) \\
& =(s \circ \pi)_{\mathcal{M}_{N}}(\underline{z})+g(\pi(\underline{z})) \cdot\left(\sum_{j \in M} q_{j}\left(z_{j}\right)\right)
\end{aligned}
$$




$$
\begin{aligned}
& =(s \circ \pi)_{\mathcal{M}_{N}}(\underline{z})+h(\underline{z}) \cdot\left(\sum_{j \in N} q_{j}\left(z_{j}\right)\right) \\
& =(s \circ \pi)_{\mathcal{M}_{N}}(\underline{z})+h(\underline{z}) \cdot\left(\sum_{j \in N}\left(q_{j}\right)_{\mathcal{M}_{N}}(\underline{z})\right) .
\end{aligned}
$$

Theorem 9.5. For every $\phi \in \mathcal{F}_{M}$ we have

$$
\phi\left(\underline{A}_{M}\right)=(\phi \circ \pi)\left(\underline{A}_{N}\right)
$$

where $\phi \circ \pi$ is as in Lemma $9.2, \phi\left(\underline{A}_{M}\right)$ is as in Definition 6.3 defined for the tuple $\underline{A}_{M}$ and $(\phi \circ \pi)\left(\underline{A}_{N}\right)$ is as in Definition 6.3 defined for the tuple $\underline{A}_{N}$.

Proof. Let $s, g$ be an admissible decomposition of $\phi \in \mathcal{F}_{M}$. By Definition 6.3 we have

$$
\phi\left(\underline{A}_{M}\right)=s\left(\underline{A}_{M}\right)+\Xi_{M}\left(\int_{\sigma\left(\Theta_{M}\left(\underline{A}_{M}\right)\right)} g d F\right),
$$

where $F$ denotes the common spectral measure of $\Theta_{M}\left(\underline{A}_{M}\right)$ as in Theorem 2.2. By Theorem 2.5 we have $F(\Delta)=E_{M}\left(\pi^{-1}(\Delta)\right)$ for Borel-subsets $\Delta \subseteq \mathbb{C}^{M}$ where $E_{M}$ denotes the common spectral measure of $\Theta_{M}\left(\underline{A}_{N}\right)$. Together with (3.9) we derive

$$
\begin{aligned}
\phi\left(\underline{A}_{M}\right) & =s\left(\underline{A}_{M}\right)+\Xi_{M}\left(\int g \circ \pi d E_{M}\right) \\
& =s\left(\underline{A}_{M}\right)+\Xi_{N}\left(R_{M / N} R_{M / N}^{*} \int g \circ \pi d E_{N}\right),
\end{aligned}
$$

where $E_{N}$ denotes the common spectral measure of $\Theta_{N}\left(\underline{A}_{N}\right)$.

Since $g \circ \pi$ vanishes on $Z_{\underline{q}_{N}}$, we have $\int g \circ \pi d E_{N}=\int_{\mathbb{C}^{n} \backslash Z_{\underline{q}_{N}}} g \circ \pi d E_{N}$. According to (4.3) we obtain

$$
\phi\left(\underline{A}_{M}\right)=(s \circ \pi)\left(\underline{A}_{N}\right)+\Xi_{N}\left(\int g(\pi(\underline{z})) \cdot \frac{\sum_{j \in N} q_{j}\left(z_{j}\right)}{\sum_{j \in M} q_{j}\left(z_{j}\right)} \cdot d E_{N}(\underline{z})\right) .
$$

According to Corollary 9.4 and Definition 6.3 this expression coincides with $(\phi \circ \pi)\left(\underline{A}_{N}\right)$.

\section{Acknowledgements}

Open access funding provided by TU Wien (TUW). The authors thank the reviewer of the present paper for making them aware of the works $[2,3]$. If the results therein had been known before, the results from [6] related to the *-algebra homomorphism $\Theta$ could have been stated and derived in an easier and more elegant manner. In fact, the part from [6] which is concerned with this homomorphism can be viewed as an alternative approach to some of the results in $[2,3]$. Nevertheless, we kept on using [6] as a basis for the present paper also after having received the reviewers report since the formulation of the results therein better matches our demands here. Moreover, since the connection of $[2,3,6]$ is not the central focus point of the present paper and 
since a detailed discussion of this connection here would result in some sort of foreign body, we refrain from including such a detailed discussion.

Open Access. This article is licensed under a Creative Commons Attribution 4.0 International License, which permits use, sharing, adaptation, distribution and reproduction in any medium or format, as long as you give appropriate credit to the original author(s) and the source, provide a link to the Creative Commons licence, and indicate if changes were made. The images or other third party material in this article are included in the article's Creative Commons licence, unless indicated otherwise in a credit line to the material. If material is not included in the article's Creative Commons licence and your intended use is not permitted by statutory regulation or exceeds the permitted use, you will need to obtain permission directly from the copyright holder. To view a copy of this licence, visit http:// creativecommons.org/licenses/by/4.0/.

Publisher's Note Springer Nature remains neutral with regard to jurisdictional claims in published maps and institutional affiliations.

\section{References}

[1] Birman, M.S., Solomjak, M.Z.: Spectral Theory of Self-adjoint Operators in Hilbert Space Mathematics and Its Applications (Soviet Series). D. Reidel Publishing Co., Dordrecht (1987)

[2] Constantinescu, T., Gheondea, A.: Representations of Hermitian kernels by means of Krein spaces. Publ. Res. Inst. Math. Sci. 33(6), 917-951 (1997)

[3] Dijksma, A., Langer, H., de Snoo, H.: Unitary Colligations in Kreĭn Spaces and Their Role in the Extension Theory of Isometries and Symmetric Linear Relations in Hilbert Spaces Functional Analysis, II (Dubrovnik, 1985), 1-42, Lecture Notes in Math., vol. 1242, pp. 1-42. Springer, Berlin (1987)

[4] Kaltenbäck, M.: Functional Calculus for definitizable normal linear operators on Krein spaces. Integral Equ. Oper. Theory 85(2), 221-243 (2016)

[5] Kaltenbäck, M.: Definitizability of normal operators on Krein spaces and their functional calculus. Integral Equ. Oper. Theory 87(4), 461-490 (2017)

[6] Kaltenbäck, M., Pruckner, R.: Functional calculus for definitizable self-adjoint linear relations on Krein spaces. Integral Equ. Oper. Theory 83(4), 451-482 (2015)

[7] Langer, H.: Spectral Functions of Definitizable Operators in Krein Spaces. Lecture Notes in Mathematics, Vol. 948, pp. 1-46 (1982)

[8] Schmüdgen, K.: Unbounded Self-adjoint Operators on Hilbert Space. Graduate Texts in Mathematics, vol. 265. Springer, Dordrecht (2012)

Michael Kaltenbäck $(\bowtie)$

Technische Universitat Wien

Wiedner Hauptstr. 8-10

1040 Vienna

Austria

e-mail: michael.kaltenbaeck@tuwien.ac.at 
Nathanael Skrepek

University of Wuppertal

Siegfriedstraße 36

42117 Wuppertal

Germany

e-mail: nathanael.skrepek@hotmail.com

Received: July 18, 2019.

Revised: June 4, 2020. 